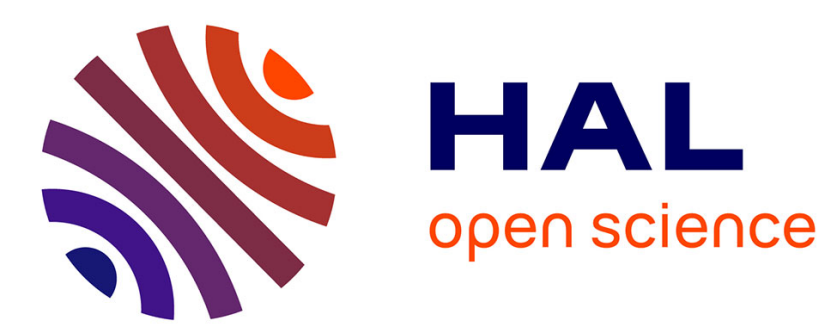

\title{
Kinetic modelling of evaporation and condensation phenomena around a spherical droplet
}

Vinícius Braga Leite, Denize Kalempa, Irina Martin Graur

\section{To cite this version:}

Vinícius Braga Leite, Denize Kalempa, Irina Martin Graur. Kinetic modelling of evaporation and condensation phenomena around a spherical droplet. International Journal of Heat and Mass Transfer, 2020, pp.120719. 10.1016/j.ijheatmasstransfer.2020.120719 . hal-03103092

\section{HAL Id: hal-03103092 \\ https://hal-amu.archives-ouvertes.fr/hal-03103092}

Submitted on 7 Jan 2021

HAL is a multi-disciplinary open access archive for the deposit and dissemination of scientific research documents, whether they are published or not. The documents may come from teaching and research institutions in France or abroad, or from public or private research centers.
L'archive ouverte pluridisciplinaire HAL, est destinée au dépôt et à la diffusion de documents scientifiques de niveau recherche, publiés ou non, émanant des établissements d'enseignement et de recherche français ou étrangers, des laboratoires publics ou privés. 


\title{
Kinetic modelling of evaporation and condensation phenomena around a spherical droplet
}

\author{
Vinícius Braga Leite ${ }^{\mathrm{a}}$, Denize Kalempa ${ }^{\mathrm{a}}$, Irina Graur ${ }^{\mathrm{b}}$ \\ ${ }^{a}$ Departamento de Ciências Básicas e Ambientais, Escola de Engenharia de Lorena, \\ Universidade de São Paulo, 12602-810, Lorena, Brazil \\ ${ }^{b}$ Aix-Marseille Université, CNRS, IUSTI UMR 7343, 13453 Marseille, France
}

\begin{abstract}
The steady evaporation and condensation phenomena around a spherical droplet of the condensed phase of a vapor are investigated with basis on a kinetic model to the linearized Boltzmann equation. The kinetic equation is solved via a discrete velocity method which takes into account the discontinuity of the distribution function of molecular velocities on the spherical interface. The calculations are carried out in a wide range of the gas rarefaction and evaporation-condensation coefficient. The results obtained via solution of the linearized Navier-Stokes equations with temperature and pressure jump boundary conditions are also presented and compared to those obtained via kinetic equation. A comparison between the linearized and nonlinear solutions of the kinetic model is also presented to show the limit of applicability of the linearized approach.
\end{abstract}

\section{Introduction}

The evaporation and condensation phenomena are of great interest in many fields such as environmental sciences, micro and nanotechnology, security of nuclear plants, etc. For instance, evaporation and condensation processes in mini and microchannels have been widely used in air-cooled condensers, in heat pipes and other devices for thermal control of different systems. In particular, microchannel condensers [1] are used to increase performance in heat transfer, to reduce components size and improve energy efficiency. The authors of Ref. [2] state that innovative cooling technologies must necessarily use the processes of boiling, evaporation and condensation in novel reduced geometrical configurations intended for the heat transfer enhancement. Moreover, according to Ref. [3], a big gap still exists between the chip-level heat generation and the system-level heat removal in high performance electronic systems, such as computers, electronics and solid-state 
lasers. Thus, one can say that evaporation and condensation phenomena play an important role in the development of saving energy and environmentally friendly new technologies.

The theory of evaporation and condensation has its foundations in the classical kinetic theory of gases. Hertz [4] and Knudsen [5] provided the molecular basis for understanding these phenomena in the free molecular flow regime, where the intermolecular collisions are negligible. Later, Pao [6, 7] and Thomas et al. [8] took into account the effect of the intermolecular collisions. These authors predicted theoretically the discontinuity of the pressure and temperature profiles through a liquid-vapor interface and explained this phenomenon by the non-equilibrium state of a gas inside the thin layer around the interface, the Knudsen layer. Labuntsov and coworkers also contributed for the development of the kinetic theory of evaporation and condensation inside the Knudsen layer, see e.g. Refs. [9, 10], and proposed useful equations to describe both weak and strong evaporation and condensation processes. Experimentally, the authors of Refs. [11 13] were the first who have measured the temperature jump at the liquid-vapor interface. Later, various campaigns were undertaken to measure the temperature discontinuity at the interface under different physical conditions, e.g. Refs. [14-18]. However, all experimental data on the temperature jump are very scattered, ranging from $15 \mathrm{~K}$ to $0.1 \mathrm{~K}$.

In practice, the Navier-Stokes (NS) equations, usually valid only under equilibrium conditions, are widely used to describe phase transition phenomena. For instance, according to Ref. [19], the hydrodynamic approach, which is usually used in engineering models of diesel fuel droplet evaporation and incorporated in relevant computational fluid dynamic codes, provides results in disagreement with realistic diesel engines, so more general kinetic models need to be employed.

Theoretical approaches for the modelling of the evaporation and condensation phenomena, based on the gas kinetic theory, e.g. Refs. [20 25], confirm that the phase transition occurs only under non-equilibrium conditions and that the continuity of the thermodynamic variables, such as temperature and pressure, cannot be ensured. Most of these studies focus on the evaporation-condensation in plane geometry of one or two condensed phases and only few authors considered the evaporation from a spherical droplet [21, 24, 26]. In Ref. [26] the problem of evaporation-condensation on a spherical droplet was studied by implementing a linearized approach for a kinetic model to the Boltzmann equation, while in Ref. [24 the same problem was solved via the linearized Boltzmann equation. The asymptotic analysis for small Knudsen number was proposed in Ref. [21]. The author of Ref. [25] derived several useful correlations for the evaporation rate and in- 
terfacial heat transfer and compared them with the numerical results of [26]. The question on the limits of the applicability of the linearized kinetic type equation for the simulation of the evaporation and condensation phenomena are discussed only in the case of the plane geometry [27, 28]. In addition, the influence of the non-complete evaporation-condensation was very often ignored.

The objective of the present work is the modelling of the steady evaporation and condensation phenomena which take place on the surface of a spherical droplet of the condensed phase of a vapor on the basis of the kinetic theory. The paper is organized as follows. First, the model proposed by Shakhov [29] for the linearized Boltzmann equation, referenced here as S-model, is solved numerically by the Discrete Velocity Method (DVM) implemented to take into account the discontinuity of the distribution function of molecular velocities around a convex body, see e.g. Refs. [30, 31]. Second, the analytical solution is derived in the case of free molecular and hydrodynamic regimes. In the hydrodynamic regime, the thermodynamic analysis is used to obtain the temperature and pressure jump boundary conditions at the liquid-vapor interface. Third, the non-linear S-model kinetic equation is solved numerically by using the DVM in the velocity space and the Total Variation Diminishing (TVD) type numerical scheme [32] for the approximation of the spatial derivatives, which does not require a special treatment for the discontinuity of the distribution function on the convex droplet surface. The mass and energy flow rates as well as the macroscopic characteristics of the gas flow around the droplet due to the phase transition were calculated in a range of the gas rarefaction which covers the free molecular, transition and hydrodynamic regimes. Moreover, complete and non-complete evaporationcondensation on the liquid-vapor interface were considered by varying the evaporation-condensation coefficient. The linearized and non-linear results were compared to find the limits of applicability of the linearized approach.

\section{Statement of the problem}

Let us consider a gas flow around its spherical condensed-phase droplet of radius $R_{0}$ caused by steady evaporation and condensation at the interface. The temperature of the liquid droplet is supposed to be constant and equal to $T_{d}$. The corresponding saturation pressure, $p_{d}=p_{\text {sat }}\left(T_{d}\right)$, could be calculated by the Clausius-Clapeyron equation [33]. Far from the droplet the gas is at thermodynamic equilibrium with pressure $p_{0}$ and temperature $T_{0}$, which are different from the pressure and temperature of the droplet.

It is worth noting that the evaporation and condensation processes are time dependent so that the droplet radius $R_{0}$ and temperature $T_{d}$ are func- 
tions of time. However, according to the literature, see e.g. [34, 35], the steady state solution is valid under the following assumptions: (i) the difference between the vapor density far from the droplet and at the interface are very small so that the droplet condensation growth and evaporation depletion can be considered as quasi-stationary processes and steady state solutions can be applied; (ii) the droplet surface temperature is maintained constant by an inner heat source within the droplet volume.

The gas rarefaction is characterized by the rarefaction parameter, $\delta$, which is inversely proportional to the well known Knudsen number, and defined here as

$$
\delta=\frac{R_{0}}{\ell_{0}}, \quad \ell_{0}=\frac{\mu_{0} v_{0}}{p_{0}}, \quad v_{0}=\sqrt{\frac{2 k_{\mathrm{B}} T_{0}}{m}}
$$

where $\ell_{0}$ is the equivalent molecular free path, $v_{0}$ is the most probable molecular velocity, $k_{\mathrm{B}}$ is the Boltzmann constant and $m$ is the molecular mass of the gas particles. In case of rigid-spheres model, the viscosity of a gas, $\mu$, is related to the viscosity $\mu_{0}$ at the equilibrium temperature $T_{0}$ as follows

$$
\mu(T)=\mu_{0} \sqrt{\frac{T}{T_{0}}}
$$

Note that the limit $\delta \rightarrow 0$ corresponds to the free molecular regime, while the limit $\delta \rightarrow \infty$ corresponds to the hydrodynamic or continuum limit.

In following we apply the S-model kinetic equation [29] to simulate the behavior of the spherical condensed-phase droplet due to the evaporation and condensation at the spherical surface. The kinetic equation is solved numerically in its linearized and non-linear forms and the obtained results are compared to establish the limits of applicability of the linearized approach. The analytical solution obtained from the linearized equations of continuum mechanics with appropriate temperature and pressure jump boundary conditions is also presented and compared to the numerical solution of the linearized model kinetic equation.

\section{Kinetic equation}

Considering the symmetry of the problem relatively to the center point, the kinetic model proposed by Shakhov [29] for the Boltzmann equation in the absence of external forces reads

$$
\frac{\partial f}{\partial t^{\prime}}+v_{r} \frac{\partial f}{\partial r^{\prime}}-\frac{v_{t}}{r^{\prime}} \frac{\partial f}{\partial \theta}=\nu\left(f^{S}-f\right),
$$


where $f\left(t^{\prime}, r^{\prime}, \mathbf{v}\right)$ is the distribution function of molecular velocities, $t^{\prime}$ is the time, $r^{\prime}$ is the radial coordinate, and $\mathbf{v}=\left(v_{r}, v_{\Theta}, v_{\Phi}\right)$ is the molecular velocity vector, where $v_{r}, v_{\Theta}$, and $v_{\Phi}$ are the radial, polar and azimuthal components in spherical coordinates $\left(v_{p}, \theta, \phi\right)$. Thus, in Eq. (3), $v_{r}=v_{p} \cos \theta$ and $v_{t}=v_{p} \sin \theta$ denote, respectively, the radial and tangential components of the molecular velocity vector $\mathbf{v}$. Note that, $v_{p}=\sqrt{v_{r}^{2}+v_{t}^{2}}$ is the magnitude of the molecular velocity vector, while $\theta$ and $\phi$ are its orientational angles. The quantity $\nu$ is the molecular collision frequency which is assumed to be independent of the molecular velocity and evaluated as

$$
\nu=\frac{p}{\mu}
$$

where $\mu$, given in (2), is the viscosity of the gas at an arbitrary temperature $T$. The Shakhov equilibrium distribution function $f^{S}$ reads

$$
\begin{aligned}
f^{S}\left(n, T, \mathbf{V}, \mathbf{q}^{\prime}\right) & =f^{M}\left[1+\frac{2 m \mathbf{V} \mathbf{q}^{\prime}}{15 n\left(k_{\mathrm{B}} T\right)^{2}}\left(\frac{m \mathbf{V}^{2}}{2 k_{\mathrm{B}} T}-\frac{5}{2}\right)\right], \\
f^{M}(n, T, \mathbf{V}) & =n\left(\frac{m}{2 \pi k_{\mathrm{B}} T}\right)^{3 / 2} \exp \left(-\frac{m \mathbf{V}^{2}}{2 k_{\mathrm{B}} T}\right),
\end{aligned}
$$

where $f^{M}$ is the local Maxwellian distribution function; $\mathbf{V}=\mathbf{v}-\mathbf{u}^{\prime}$ is the peculiar velocity vector, $\mathbf{u}^{\prime}=\left(u_{r}^{\prime}, 0,0\right)$ is the bulk velocity vector; $\mathbf{q}^{\prime}=\left(q_{r}^{\prime}, 0,0\right)$ is the heat flux vector.

The macroscopic quantities corresponding to the gas number density, the temperature, the bulk velocity and heat flux vectors, are calculated in terms of the distribution function of molecular velocities, see e.g. Refs. [36, 37], as follows

$$
\begin{aligned}
& n\left(t^{\prime}, r^{\prime}\right)=\int_{0}^{\infty} \int_{0}^{\pi} \int_{0}^{2 \pi} f\left(t^{\prime}, r^{\prime}, \mathbf{v}\right) \mathrm{d} \mathbf{v}, \\
& T\left(t^{\prime}, r^{\prime}\right)=\frac{m}{3 n k_{\mathrm{B}}} \int_{0}^{\infty} \int_{0}^{\pi} \int_{0}^{2 \pi} V^{2} f\left(t^{\prime}, r^{\prime}, \mathbf{v}\right) \mathrm{d} \mathbf{v}, \\
& \mathbf{u}^{\prime}\left(t^{\prime}, r^{\prime}\right)=\frac{1}{n} \int_{0}^{\infty} \int_{0}^{\pi} \int_{0}^{2 \pi} \mathbf{v} f\left(t^{\prime}, r^{\prime}, \mathbf{v}\right) \mathrm{d} \mathbf{v}, \\
& \mathbf{q}^{\prime}\left(t^{\prime}, r^{\prime}\right)=\frac{m}{2} \int_{0}^{\infty} \int_{0}^{\pi} \int_{0}^{2 \pi} V^{2} \mathbf{v} f\left(t^{\prime}, r^{\prime}, \mathbf{v}\right) \mathrm{d} \mathbf{v},
\end{aligned}
$$


where $\mathrm{d} \mathbf{v}=v_{p}^{2} \sin \theta \mathrm{d} v_{p} \mathrm{~d} \theta \mathrm{d} \phi$. The mass and energy flow rates, which are the quantities of practical interest, are also calculated in terms of the distribution function of molecular velocities as

$$
\begin{aligned}
& \dot{M}^{\prime}=\int_{R_{0}}^{\infty} \mathrm{d} A^{\prime} \int_{0}^{\infty} \int_{0}^{\pi} \int_{0}^{2 \pi} m \mathbf{v} f\left(t^{\prime}, r^{\prime}, \mathbf{v}\right) \mathrm{d} \mathbf{v}, \\
& \dot{E}^{\prime}=\int_{R_{0}}^{\infty} \mathrm{d} A^{\prime} \int_{0}^{\infty} \int_{0}^{\pi} \int_{0}^{2 \pi} \mathbf{v} \frac{m v^{2}}{2} f\left(t^{\prime}, r^{\prime}, \mathbf{v}\right) \mathrm{d} \mathbf{v},
\end{aligned}
$$

where $\mathrm{d} A^{\prime}=8 \pi r^{\prime} \mathrm{d} r^{\prime}$ is an area element of a spherical surface with radius $r^{\prime}$.

On the droplet surface we assume that the fraction $\sigma$ of incident molecules condensates at the surface and then evaporates from it with the equilibrium Maxwellian distribution function, while the fraction $1-\sigma$ is reflected under the assumption of diffuse scattering. Therefore, the boundary condition at the vapor-liquid interface is written as

$$
f\left(t, R_{0}, \mathbf{v}\right)=\sigma f_{s}+(1-\sigma) f_{r} \quad \text { at } \quad v_{r}>0,
$$

where $f_{s}$ and $f_{r}$ are the equilibrium Maxwellian functions given by

$$
f_{i}=n_{i}\left(\frac{m}{2 \pi k_{\mathrm{B}} T_{s}}\right)^{3 / 2} \exp \left(-\frac{m v_{p}^{2}}{2 k_{\mathrm{B}} T_{s}}\right), \quad i=s, r,
$$

where $n_{d}$ is the number density calculated from equation of state using the droplet temperature and pressure as $n_{d}=p_{d} /\left(k_{\mathrm{B}} T_{d}\right)$ and $n_{r}$ is the number density calculated from the impermeability condition for the molecules reflected diffusively from the surface.

Far from the sphere the gas is assumed to be in thermodynamic equilibrium so that

$$
f_{\infty}=\lim _{r^{\prime} \rightarrow \infty} f\left(t^{\prime}, r^{\prime}, \mathbf{v}\right)=f_{0}^{M}
$$

where the Maxwellian distribution function is given by

$$
f_{0}^{M}=n_{0}\left(\frac{m}{2 \pi k_{\mathrm{B}} T_{0}}\right)^{3 / 2} \exp \left(-\frac{m v_{p}^{2}}{2 k_{\mathrm{B}} T_{0}}\right),
$$

where the number density $n_{0}$ is calculated from the equation of state $n_{0}=$ $p_{0} /\left(k_{\mathrm{B}} T_{0}\right)$.

In the numerical calculations, a maximum radial distance from the centre of the spherical droplet is set to ensure the thermodynamic equilibrium far from the sphere. 
It is worth mentioning that, although our problem concerns the steady evaporation and condensation at the vapor-liquid interface, the time dependence of the distribution function was maintained in this section, essentially because the numerical method used for the solution of the non-linear kinetic equation is based on the iteration in time, see Section 5.2. The implicit numerical scheme is adopted here to decrease the computational time. However, the explicit scheme could be also applied to quantify the evaporation rate in time.

\section{Linearized approach}

\subsection{Linearized kinetic equation}

To simulate the evaporation condensation phenomena, two thermodynamic driven forces are introduced as follows

$$
X_{n}=\frac{\left|n_{d}-n_{0}\right|}{n_{0}}, \quad X_{T}=\frac{\left|T_{d}-T_{0}\right|}{T_{0}} .
$$

Since the saturation pressure depends on the saturation temperature, these two thermodynamic forces are not independent quantities and could be related by the Clausius-Clapeyron equation. However, in the frame of this study, these forces are assumed as being independent from each other, to see clearly the impact of each force on the evaporation process.

When the system is weakly disturbed from thermodynamic equilibrium, the thermodynamic forces given in (16) are very small, i.e. $X_{n} \ll 1$ and $X_{T} \ll 1$. Therefore, the kinetic equation (3) can be linearized by representing the distribution function of molecular velocities as

$$
f\left(r^{\prime}, \mathbf{v}\right)=f_{0}^{M}\left[1+h^{(n)}\left(r^{\prime}, \mathbf{v}\right) X_{n}+h^{(T)}\left(r^{\prime}, \mathbf{v}\right) X_{T}\right],
$$

where the Maxwellian distribution function corresponding to the gas equilibrium state far from the spherical droplet is given by (15). The functions $h^{(n)}$ and $h^{(T)}$ are the perturbation functions due to the density and the temperature deviations, $X_{n}$ and $X_{T}$, respectively. For further derivations, the dimensionless quantities corresponding to the radial coordinate $r$, molecular velocity vector $\mathbf{c}=\left(c_{r}, c_{\Theta}, c_{\Phi}\right)$, bulk velocity vector $\mathbf{u}=\left(u_{r}, 0,0\right)$ and heat flux vector $\mathbf{q}=\left(q_{r}, 0,0\right)$ are introduced as

$$
r=\frac{r^{\prime}}{R_{0}}, \quad \mathbf{c}=\frac{\mathbf{v}}{v_{0}}, \quad \mathbf{u}=\frac{\mathbf{u}^{\prime}}{v_{0}}, \quad \mathbf{q}=\frac{\mathbf{q}^{\prime}}{p_{0} v_{0}} .
$$

Thus, the representation (17) allows us to write the macroscopic characteristics of the gas flow given in (6)-(9) as follows

$$
\begin{aligned}
& n=n_{0}\left(1+\nu^{(n)} X_{n}+\nu^{(T)} X_{T}\right), \quad T=T_{0}\left(1+\tau^{(n)} X_{n}+\tau^{(T)} X_{T}\right), \\
& \mathbf{u}=\mathbf{u}^{(n)} X_{n}+\mathbf{u}^{(T)} X_{T}, \quad \mathbf{q}=\mathbf{q}^{(n)} X_{n}+\mathbf{q}^{(T)} X_{T} .
\end{aligned}
$$


Moreover, the substitution of the representation (17) into the kinetic equation (3) allows us to obtain two independent linearized kinetic equations for each thermodynamic force which read

$$
\begin{aligned}
& c_{r} \frac{\partial h^{(i)}}{\partial r}-\frac{c_{t}}{r} \frac{\partial h^{(i)}}{\partial \theta}=\delta\left[\nu^{(i)}+\left(c^{2}-\frac{3}{2}\right) \tau^{(i)}\right. \\
& \left.\quad+2 c_{r} u_{r}^{(i)}+\frac{4}{15} c_{r}\left(c^{2}-\frac{5}{2}\right) q_{r}^{(i)}-h^{(i)}\right], \quad i=n, T,
\end{aligned}
$$

where $c_{r}=c_{p} \cos \theta$ and $c_{t}=c_{p} \sin \theta$ denote the dimensionless radial and tangential components of the molecular velocity vector $\mathbf{c}$. The dimensionless moments appearing on the right hand side of Eq. (20) corresponding to each thermodynamic force are obtained from $(19)$ and $(6)-(9)$ as

$$
\begin{aligned}
& \nu^{(i)}(r)=\frac{2}{\sqrt{\pi}} \int_{0}^{\infty} \int_{0}^{\pi} h^{(i)}\left(r, \theta, c_{p}\right) \mathrm{e}^{-c_{p}^{2}} \mathrm{~d} \mathbf{c}, \\
& \tau^{(i)}(r)=\frac{4}{3 \sqrt{\pi}} \int_{0}^{\infty} \int_{0}^{\pi}\left(c^{2}-\frac{3}{2}\right) h^{(i)}\left(r, \theta, c_{p}\right) \mathrm{e}^{-c_{p}^{2}} \mathrm{~d} \mathbf{c}, \\
& u_{r}^{(i)}(r)=\frac{2}{\sqrt{\pi}} \int_{0}^{\infty} \int_{0}^{\pi} c_{p} \cos \theta h^{(i)}\left(r, \theta, c_{p}\right) \mathrm{e}^{-c_{p}^{2}} \mathrm{~d} \mathbf{c}, \\
& q_{r}^{(i)}(r)=\frac{2}{\sqrt{\pi}} \int_{0}^{\infty} \int_{0}^{\pi} c_{p} \cos \theta\left(c^{2}-\frac{5}{2}\right) h^{(i)}\left(r, \theta, c_{p}\right) \mathrm{e}^{-c_{p}^{2}} \mathrm{~d} \mathbf{c},
\end{aligned}
$$

where $\mathrm{d} \mathbf{c}=c_{p}^{2} \sin \theta \mathrm{d} c_{p} \mathrm{~d} \theta$. For convenience, hereafter, the subscript " $r$ " denoting the radial components of the gas bulk velocity and heat flux is omitted.

From the boundary condition (12), the representation (17) leads to the following linearized boundary conditions for the perturbation functions due to $X_{n}$ and $X_{T}$

$$
\begin{aligned}
& h^{(n)}\left(1, \theta, c_{p}\right)=\sigma+(1-\sigma) n_{r}^{(n)}, \quad \text { at } \quad c_{r}>0, \\
& h^{(T)}\left(1, \theta, c_{p}\right)=\left(c^{2}-\frac{3}{2}\right)+(1-\sigma) n_{r}^{(T)} \quad \text { at } \quad c_{r}>0,
\end{aligned}
$$


where the parameters $n_{r}^{(n)}$ and $n_{r}^{(T)}$ are obtained from the impermeability condition for the gas at the interface as

$$
n_{r}^{(n)}=-4 \int_{0}^{\infty} \int_{\pi / 2}^{\pi} c_{p}^{3} h^{(n)}\left(1, \theta, c_{p}\right) \sin \theta \cos \theta \mathrm{d} c_{p} \mathrm{~d} \theta
$$

and

$$
n_{r}^{(T)}=-\frac{1}{2}-4 \int_{0}^{\infty} \int_{\pi / 2}^{\pi} c_{p}^{3} h^{(T)}\left(1, \theta, c_{p}\right) \sin \theta \cos \theta \mathrm{d} c_{p} \mathrm{~d} \theta .
$$

Far from the sphere, the substitution of the representation (17) into (14) leads to

$$
h_{\infty}^{(i)}=\lim _{r \rightarrow \infty} h^{(i)}=0, \quad i=n, T .
$$

The infinite interval $[0, \infty)$ for the radial distance $r$ is substituted by a finite interval $\left[0, r_{\max }\right]$, where the maximum distance $r_{\max }$ must be chosen to guarantee the thermodynamic equilibrium far from the spherical droplet.

\subsection{Thermodynamic analysis}

From the mass and energy conservation laws we can show that the dimensionless mass and energy flow rates read

$$
\begin{aligned}
& \dot{M}=\frac{\dot{M}^{\prime}}{4 \pi R_{0}^{2} m n_{0} v_{0}}=r^{2} u, \\
& \dot{E}=\frac{\dot{E}^{\prime}}{4 \pi R_{0}^{2} v_{0} p_{0}}=r^{2}\left(q+\frac{5}{2} u\right),
\end{aligned}
$$

where $\dot{M}^{\prime}$ and $\dot{E}^{\prime}$ are defined in $(10)$ and $(11)$. Note that the quantities on the right hand side of (30) and (31) are constant, i.e. they do not depend on the radial coordinate.

According to the thermodynamics of irreversible processes [38], the entropy production $S$ can be written as

$$
S=k_{\mathrm{B}}\left[J_{n}^{\prime} X_{n}+\left(J_{n}^{\prime}+J_{T}^{\prime}\right) X_{T}\right],
$$

where the thermodynamic fluxes are defined as

$$
J_{n}^{\prime}=\int n_{0} v_{0} u \mathrm{~d} A^{\prime}, \quad J_{T}^{\prime}=\int n_{0} v_{0} q \mathrm{~d} A^{\prime}
$$


and the thermodynamic forces $X_{n}$ and $X_{T}$ are defined in (16). These thermodynamic fluxes satisfy the linear relations

$$
\begin{aligned}
& J_{n}^{\prime}=\Lambda_{n n}^{\prime} X_{n}+\Lambda_{n T}^{\prime} X_{T}, \\
& J_{n}^{\prime}+J_{T}^{\prime}=\Lambda_{T n}^{\prime} X_{n}+\Lambda_{T T}^{\prime} X_{T},
\end{aligned}
$$

where $\Lambda_{i j}$ are the so called kinetic coefficients which satisfy the Onsager reciprocity relation $\Lambda_{n T}=\Lambda_{T n}$. Thus, the mass and energy flow rates defined in (10) and (11) can be written in terms of the thermodynamic fluxex $J_{n}^{\prime}$ and $J_{T}^{\prime}$ as

$$
\dot{M}^{\prime}=m J_{n}^{\prime}, \quad \dot{E}^{\prime}=k_{\mathrm{B}} T\left(J_{T}^{\prime}+\frac{5}{2} J_{n}^{\prime}\right)
$$

The advantage of using $J_{n}^{\prime}$ and $J_{T}^{\prime}$ instead of $\dot{M}^{\prime}$ and $\dot{E}^{\prime}$ is that the number of independent kinetic coefficients is reduced from four to three due to the reciprocity relation. Moreover, the reciprocity relation represents an important criterion to verify the accuracy of the numerical calculations. For convenience, the dimensionless kinetic coefficients are introduced as

$$
\Lambda_{i j}=\frac{\Lambda_{i j}^{\prime}}{4 \pi R_{0}^{2} n_{0} v_{0}}, \quad i, j=n, T
$$

and are obtained from (10), (11), (34), (35) and (36) as

$$
\begin{aligned}
& \Lambda_{n n}=r^{2} u^{(n)}, \quad \Lambda_{n T}=r^{2} u^{(T)}, \\
& \Lambda_{T n}=r^{2}\left(u^{(n)}+q^{(n)}\right), \quad \Lambda_{T T}=r^{2}\left(u^{(T)}+q^{(T)}\right) .
\end{aligned}
$$

Therefore, the reciprocity relation for the problem in question reads

$$
u^{(T)}(r)=u^{(n)}(r)+q^{(n)}(r) .
$$

\subsection{Analytic solution in the free molecular regime}

When $\delta \ll 1$, the intermolecular collisions can be neglected and, consequently, the linearized kinetic equations given in (20) are reduced to

$$
c_{r} \frac{\partial h^{(i)}}{\partial r}-\frac{c_{t}}{r} \frac{\partial h^{(i)}}{\partial \theta}=0, \quad i=n, T .
$$


Moreover, in this flow regime, the perturbation function of the incident particles at the interface corresponds to that far from the sphere, given by (29). As a consequence, the boundary conditions given by (25) and (26) are reduced to

$$
\begin{aligned}
& h^{(n)}\left(1, \theta, c_{p}\right)=\sigma \quad \text { at } \quad c_{r}>0, \\
& h^{(T)}\left(1, \theta, c_{p}\right)=c^{2}-2+\frac{\sigma}{2} \text { at } c_{r}>0 .
\end{aligned}
$$

The kinetic equations given in (41) can be solved analytically by employing the known method of the characteristics to solve a differential equation. Thus, the solution of Eq. (41) subject to the boundary condition corresponding to each thermodynamic force, given by (42) and (43), reads

$$
\begin{aligned}
& h^{(n)}\left(r, \theta, c_{p}\right)= \begin{cases}\sigma, & 0 \leq \theta \leq \theta_{0}, \\
0, & \theta_{0}<\theta \leq \pi,\end{cases} \\
& h^{(T)}\left(r, \theta, c_{p}\right)= \begin{cases}c^{2}-2+\frac{\sigma}{2}, \quad 0 \leq \theta \leq \theta_{0}, \\
0, & \theta_{0}<\theta \leq \pi,\end{cases}
\end{aligned}
$$

where the angle $\theta_{0}$ is given by

$$
\theta_{0}=\arcsin \left(\frac{1}{r}\right)
$$

After substituting the solutions (44) and (45) into (21)-(24), the following expressions for the macroscopic characteristics of the gas flow are obtained

$$
\begin{gathered}
\nu^{(n)}(r)=\frac{\sigma}{2}\left[1-\sqrt{1-\left(\frac{1}{r}\right)^{2}}\right], \quad \tau^{(n)}(r)=0 \\
\nu^{(T)}(r)=-\frac{(1-\sigma)}{4}\left[1-\sqrt{1-\left(\frac{1}{r}\right)^{2}}\right], \tau^{(T)}(r)=\frac{1}{2}\left[1-\sqrt{1-\left(\frac{1}{r}\right)^{2}}\right], \\
u^{(n)}(r)=\frac{\sigma}{2 \sqrt{\pi}}\left(\frac{1}{r}\right)^{2}, \quad u^{(T)}(r)=\frac{\sigma}{4 \sqrt{\pi}}\left(\frac{1}{r}\right)^{2}
\end{gathered}
$$




$$
q^{(n)}(r)=-\frac{\sigma}{4 \sqrt{\pi}}\left(\frac{1}{r}\right)^{2}, \quad q^{(T)}(r)=\frac{1}{\sqrt{\pi}}\left(1-\frac{\sigma}{8}\right)\left(\frac{1}{r}\right)^{2} .
$$

The dimensionless mass flow rate at the interface is obtained from 30 as

$$
\dot{M}=\frac{\sigma}{2 \sqrt{\pi}} X_{n}+\frac{\sigma}{4 \sqrt{\pi}} X_{T}
$$

and corresponds to widely used Hertz-Knudsen formula for the mass flow rate of vapor from the droplet. The energy flow rate is obtained from (31) as

$$
\dot{E}=\frac{\sigma}{\sqrt{\pi}} X_{n}+\frac{1}{\sqrt{\pi}}\left(1+\frac{\sigma}{2}\right) X_{T}
$$

\subsection{Numerical solution for arbitrary gas rarefaction}

The linearized kinetic equations given in (20) subject to the corresponding boundary condition for each thermodynamic force are solved numerically by applying the DVM, whose details can be found in the literature, see e.g. Ref. [39]. Moreover, the split method proposed in Ref. [40] to deal with the problem of the discontinuity of the distribution function of molecular velocities on the spherical interface is employed. In rarefied gas dynamics, the problem of the discontinuity of the distribution function is a peculiarity inherent to gas flows around convex bodies, see e.g. Ref. [41], and must be treated carefully when a finite difference scheme is used. According to the analytic solutions in the free molecular regime, given in (44) and (45), the discontinuity occurs when $\theta=\theta_{0}$. For small values of rarefaction parameter this behavior still remains for $\theta=\theta_{0}$ and, as a consequence, the numerical solution based on the finite difference scheme to calculate the derivative $\partial h / \partial \theta$ leads to a significant numerical error. The split method consists on the decomposition of the perturbation function into two parts as

$$
h^{(i)}\left(r, \theta, c_{p}\right)=h_{0}^{(i)}\left(r, \theta, c_{p}\right)+\tilde{h}^{(i)}\left(r, \theta, c_{p}\right), \quad i=n, T,
$$

where $h_{0}^{(i)}$ satisfy the following partial differential equation

$$
c_{r} \frac{\partial h_{0}^{(i)}}{\partial r}-\frac{c_{t}}{r} \frac{\partial h_{0}^{(i)}}{\partial \theta}+\delta h_{0}^{(i)}=0
$$

subject to the boundary conditions

$$
h_{0}^{(n)}\left(1, \theta, c_{p}\right)=\sigma \quad \text { at } \quad c_{r}>0,
$$




$$
h_{0}^{(T)}\left(1, \theta, c_{p}\right)=c^{2}-2+\frac{\sigma}{2} \quad \text { at } \quad c_{r}>0
$$

The functions $\tilde{h}^{(n)}$ and $\tilde{h}^{(T)}$ satisfy the kinetic equations given by 20 , just changing $h^{(i)}$ by $\tilde{h}^{(i)}(i=n, T)$, with boundary conditions

$$
\tilde{h}^{(i)}\left(1, \theta, c_{p}\right)=(1-\sigma) \tilde{n}_{r}^{(i)} \quad \text { at } \quad c_{r}>0,
$$

where

$$
\tilde{n}_{r}^{(i)}=\int_{0}^{\infty} \int_{\pi / 2}^{\pi} c^{3} \tilde{h}^{(i)}\left(1, \theta, c_{p}\right) \sin \theta \cos \theta \mathrm{d} c_{p} \mathrm{~d} \theta .
$$

The advantage of this method is that the function $h_{0}^{(i)}$ is found analytically by employing the method of the characteristics, while the function $\tilde{h}^{(i)}$ is sufficiently smooth so that a finite difference scheme can be used and it leads to a smaller numerical error. For convenience, the analytic solution $h_{0}^{(i)}$ $(i=n, T)$, for each thermodynamic force is presented in Appendix A. It is worth noting that the moments given in (21)-(24) are also decomposed into two parts due to the representation (53). Thus, they are written as

$$
\begin{array}{ll}
\nu^{(i)}(r)=\nu_{0}^{(i)}(r)+\tilde{\nu}^{(i)}(r), & \tau^{(i)}(r)=\tau_{0}^{(i)}(r)+\tilde{\tau}^{(i)}(r), \\
u^{(i)}(r)=u_{0}^{(i)}(r)+\tilde{u}^{(i)}(r), & q^{(i)}(r)=q_{0}^{(i)}(r)+\tilde{q}^{(i)}(r),
\end{array}
$$

where the quantities with tilde are calculated via the expressions given in (21)-(24), just changing $h^{(i)}$ by $\tilde{h}^{(i)}$, while the quantities with subscript "0" are calculated in terms of the function $h_{0}^{(i)}$ and their expressions are provided in Appendix A. To show the typical behavior of the perturbation functions $h^{(n)}$ and $h^{(T)}$ depending on the angle $\theta$ for small values of $\delta$ when the DVM with and without the split technique is employed, some figures are provided as online supplementary material.

The kinetic equation for each thermodynamic force subject to the corresponding boundary condition and asymptotic behavior was solved via the DVM with an accuracy of $0.1 \%$ for the gas flow macroscopic characteristics at the vapor-liquid interface. The calculations were carried out in a range of the gas rarefaction which covers the free molecular, transition and hydrodynamic flow regimes. The Gaussian-Hermite quadrature was used to discretize the molecular velocity space and calculate the moments of the perturbation function. The numerical technique to calculate the nodes and weights is described in Ref. [42]. A central finite difference scheme was used to approximate the derivatives appearing in the kinetic equation. The accuracy was estimated 
by varying the grid parameters $N_{r}, N_{\theta}$ and $N_{c}$, corresponding to the number of nodes in the radial coordinate $r$, angle $\theta$ and magnitude of the molecular velocity, $c_{p}$. The values of these parameters, $N_{c}$ and $N_{\theta}$, were fixed at 12 and 200 , respectively, while $N_{r}$ varied according to the maximum value of radial coordinate, $r_{\max }$, so that the increment $\Delta r \sim 10^{-3}$. The distance $r_{\max }$ was chosen so that the dimensional distance $r_{\max }^{\prime}$ varied from $10 \ell_{0}$ to $100 \ell_{0}$ when the rarefaction parameter varied from 0.01 to 10 . Note that, from Eqs. (1) and (18), the distance $r_{\max }^{\prime}$ can be written in terms of the equivalent mean free path $\ell_{0}$ as $r_{\text {max }}^{\prime}=r_{\max } \delta \ell_{0}$. In the limits $\delta \rightarrow 0$ and $\delta \rightarrow \infty$, the numerical solution was compared to those obtained analytically in the free molecular and hydrodynamic regimes. The reciprocal relation (40) was verified within the numerical error.

\subsection{Hydrodynamic solution}

When $\delta \gg 1$, the problem can be solved via the linearized continuum equations with appropriate pressure and temperature jump conditions at the vapor-liquid interface. Although the modelling via the kinetic equation provides more accurate information concerning the evaporation-condensation process, in this flow regime the convergence of the numerical scheme based on the discrete velocity method is very slow due to the large number of the grid points necessary to guarantee the accuracy of the calculations. Then, in this flow regime, it is more convenient to use the continuum equations. In our notation, the linearized balance equations of mass, momentum and energy read

$$
\begin{aligned}
& \frac{d\left(r^{2} u\right)}{d r}=0, \\
& \frac{d p}{d r}=0, \\
& \frac{d}{d r}\left(r^{2} \frac{d T}{d r}\right)=0,
\end{aligned}
$$

where the pressure is expressed via the equation of state of an ideal gas. The solution of these equations requires the use of appropriate boundary conditions which take into account the pressure and temperature jumps at the vapor-liquid interface. Since the processes of heat and mass transfer at the interface can be described by the non-equilibrium thermodynamics, the boundary conditions for the pressure and temperature at the interface can be 
obtained from the inverted form of the flux-force relations. Thus, according to Ref. [43], one can write

$$
\begin{aligned}
& p=p_{s}\left(1-\zeta_{\mathrm{P}} J_{n}-\zeta_{\mathrm{T}} J_{T}\right), \quad \text { at } \quad r=1, \\
& T=T_{s}\left(1-\zeta_{\mathrm{T}} J_{n}-\zeta_{\mathrm{TP}} J_{T}\right), \quad \text { at } \quad r=1,
\end{aligned}
$$

where the dimensionless thermodynamic fluxes $J_{i}(i=n, T)$ are introduced as

$$
J_{i}=\frac{J_{i}^{\prime}}{4 \pi R_{0}^{2} n_{0} v_{0}},
$$

and $J_{i}^{\prime}$ are defined in $(33)$. The jump coefficients are obtained from the kinetic theory, see e.g. Ref. [44], and in case of monoatomic gas under the assumption of diffuse reflection of the fraction of particles which do not condense at the interface read

$$
\zeta_{\mathrm{P}}=2 \sqrt{\pi}\left(\frac{1}{\sigma}-0.40044\right), \quad \zeta_{\mathrm{T}}=0.446658, \quad \zeta_{\mathrm{TP}}=1.042203 .
$$

From the balance equations $(60)-(62)$ and the vapor-liquid interface conditions (63) and (64), the bulk velocity and temperature deviation of the gas flow from equilibrium are obtained as follows

$$
\begin{aligned}
u(r) & =\frac{1}{r^{2}} \frac{\left[X_{n}+\left(1-\zeta_{\mathrm{T}} \xi\right) X_{T}\right]}{\left(\zeta_{\mathrm{P}}-\zeta_{\mathrm{T}}^{2} \xi\right)}, \\
\tau(r) & =-\frac{1}{r(1+\kappa)} \frac{\left[\zeta_{\mathrm{T}} X_{n}-\left(\zeta_{\mathrm{P}}-\zeta_{\mathrm{T}}\right) X_{T}\right]}{\left(\zeta_{\mathrm{P}}-\zeta_{\mathrm{T}}^{2} \xi\right)},
\end{aligned}
$$

where

$$
\kappa=\frac{\zeta_{\mathrm{TP}}}{2 \delta} \frac{\gamma}{(\gamma-1)} \frac{1}{\operatorname{Pr}}, \quad \xi=\frac{\kappa}{\zeta_{\mathrm{TP}}} \frac{1}{(1+\kappa)} .
$$

In the previous expression, $\gamma=c_{p} / c_{v}$ is the ratio of the specific heat capacities and $\mathrm{Pr}$ is the Prandtl number. These parameters for a monatomic gas are equal to $5 / 3$ and $2 / 3$, respectively.

According to the balance equation (61), the pressure is a constant quantity. Therefore, since the temperature profile is known from (68), the density deviation from equilibrium reads

$$
\nu(r)=-\tau(r) .
$$


The heat flux is obtained from the Fourier law as

$$
q(r)=-\frac{1}{r^{2}} \frac{\kappa}{\zeta_{\mathrm{TP}}(1+\kappa)} \frac{\left[\zeta_{\mathrm{T}} X_{n}-\left(\zeta_{\mathrm{P}}-\zeta_{\mathrm{T}}\right) X_{T}\right]}{\left(\zeta_{\mathrm{P}}-\zeta_{\mathrm{T}}^{2} \xi\right)} .
$$

Note that, in the limit $\delta \rightarrow \infty$, the expressions $(67),(68)$ and $(71)$ tend to

$$
\begin{aligned}
& u(r)=\frac{1}{\zeta_{\mathrm{P}} r^{2}}\left(X_{n}+X_{T}\right), \\
& \tau(r)=-\frac{1}{r}\left[\frac{\zeta_{\mathrm{T}}}{\zeta_{\mathrm{P}}} X_{n}-\left(1-\frac{\zeta_{\mathrm{T}}}{\zeta_{\mathrm{P}}}\right) X_{T}\right], \\
& q(r)=-\frac{1}{r^{2}} \frac{\kappa}{\zeta_{\mathrm{TP}}}\left[\frac{\zeta_{\mathrm{T}}}{\zeta_{\mathrm{P}}} X_{n}-\left(1-\frac{\zeta_{\mathrm{T}}}{\zeta_{\mathrm{P}}}\right) X_{T}\right] .
\end{aligned}
$$

The dimensionless mass and energy flow rates at the vapor-liquid interface are calculated from 30 and (31). It is worth to underline that, in our notation, the widely used Schrage relation [45] for the mass flow rate under the assumption of very small driving forces reads

$$
\dot{M}_{S c h}=\frac{\sigma}{(2-\sigma) \sqrt{\pi}}\left(X_{n}+\frac{1}{2} X_{T}\right) .
$$

Thus, in order to compare the present results with those obtained from the Schrage relation, the dimensionless mass flow rate at the interface obtained from $(30)$ and $(72)$ is used and it reads

$$
\dot{M}=\frac{1}{\zeta_{\mathrm{P}}}\left(X_{n}+X_{T}\right),
$$

The comparison between the results obtained from the continuum approach (76), the kinetic modelling and Schrage expression (75) is presented in Section 6.

\section{Non-linear approach}

\subsection{Non-linear kinetic equation and boundary conditions}

To establish the limits of applicability of the linearized approach, the non-linear S-model kinetic equation (3) is solved numerically. Additionally 
to the dimensionless quantities introduced in (18), the defined dimensionless variables are used in the following

$$
\hat{f}=\frac{f v_{0}^{3}}{n_{0}}, \quad t=\frac{t^{\prime} v_{0}}{R_{0}}, \quad \hat{n}=\frac{n}{n_{0}}, \quad \hat{T}=\frac{T}{T_{0}}, \quad \hat{\mu}=\frac{\mu}{\mu_{0}} .
$$

Thus, the dimensionless form of the kinetic equation (3) reads

$$
\frac{\partial \hat{f}}{\partial t}+c_{r} \frac{\partial \hat{f}}{\partial r}-\frac{c_{t}}{r} \frac{\partial \hat{f}}{\partial \theta}=\delta \hat{n} \sqrt{\hat{T}}\left(\hat{f}^{S}-\hat{f}\right) .
$$

The dimensionless boundary condition for solving the previous equation is obtained from 12 as

$$
\hat{f}\left(t, 1, \theta, c_{p}\right)=\sigma \hat{f}_{s}+(1-\sigma) \hat{f}_{r}, \quad c_{r}>0 .
$$

Moreover, far from the spherical droplet, the normalized distribution function satisfies the initial condition obtained from (14) as

$$
\hat{f}\left(0, r, \theta, c_{p}\right)=\hat{f}_{\infty}=\lim _{r \rightarrow \infty} \hat{f}\left(t, r, \theta, c_{p}\right)=\frac{1}{\pi^{3 / 2}} \mathrm{e}^{-c_{p}^{2}} .
$$

The flow fields given in (6)-(9) are written in terms of the dimensionless distribution function introduced here as

$$
\begin{aligned}
& \hat{n}(t, r)=2 \pi \int_{0}^{\infty} \int_{0}^{\pi} \hat{f}\left(t, r, \theta, c_{p}\right) \mathrm{d} \mathbf{c}, \\
& \hat{T}(t, r)=\frac{2 \pi}{3 n} \int_{0}^{\infty} \int_{0}^{\pi}\left[\left(c_{r}-u_{r}\right)^{2}+c_{t}^{2}\right] \hat{f}\left(t, r, \theta, c_{p}\right) \mathrm{d} \mathbf{c}, \\
& \hat{u}_{r}(t, r)=\frac{2 \pi}{n} \int_{0}^{\infty} \int_{0}^{\pi} c_{r} \hat{f}\left(t, r, \theta, c_{p}\right) \mathrm{d} \mathbf{c}, \\
& \hat{q}_{r}(t, r)=2 \pi \int_{0}^{\infty} \int_{0}^{\pi} c_{r}\left[\left(c_{r}-u_{r}\right)^{2}+c_{t}^{2}\right] \hat{f}\left(t, r, \theta, c_{p}\right) \mathrm{d} \mathbf{c} .
\end{aligned}
$$

Once again, for convenience, the subscript " $r$ " denoting the radial components of the gas bulk velocity and heat flux is omitted as well as the superscript ^ for dimensionless variables.

The dimensionless mass and energy flow rates are given by the previous expressions 30 and $(31)$. 


\subsection{Method of solution}

To obtain the flow parameters in the transitional regime, Eq. (78) is solved numerically by applying the technique developed previously in Ref. [32. First, the DVM is applied to split the continuum molecular velocity space $c_{p}$ in the governing equation (78) into discrete velocity set $c_{p_{m}}$, in which $m=1,2, . ., N_{c_{P}}$. The Gaus-Hermite quadrature is used to calculate the integrals in the velocity space $c_{p}$ appearing in the calculation of the gas flow fields, given in (81)-84). As a consequence, the velocity magnitudes $c_{p_{m}}$ are taken to be the roots of the Hermite polynomial of order $N_{c_{P}}$ and associated with their corresponding weights. Then the set of $N_{c_{P}}$ kinetic equations, corresponding to $N_{c_{P}}$ values of discrete velocity $c_{p_{m}}$, is discretized in time and space by finite difference method (FDM). The range of orientation angle $\theta$ $(0 \leq \theta \leq \pi)$ is divided into $N_{\theta}$ uniform segments defined by $\theta_{l}, l=0,1, . ., N_{\theta}$, and the integrals in the angle $\theta$ appearing in (81)-(84) are calculated by the Simpson $3 / 8$ rule.

A maximum radial coordinate, $r_{\max }$, is set and the gap between $r_{\max }$ and $r_{0}$ is divided into $N_{r}$ equal intervals characterized by $r_{i}, i=0,1, . ., N_{r}$. Let us denote the numerical solution of Eq. $(78)$ at the time level $k$ as $f_{i, l, m}^{k}=$ $f\left(t^{k}, r_{i}, \theta_{l}, c_{p_{m}}\right)$ and define $\Delta t^{k}=t^{k+1}-t^{k}, \Delta r_{i}=r_{i+1}-r_{i}, \Delta \theta_{l}=\theta_{l+1}-\theta_{l}$, $\Delta f^{k}=f_{i, l, m}^{k+1}-f_{i, l, m}^{k}$.

In this paper we are interested only in the steady-state solution, therefore the fully time-implicit Godunov-type scheme is applied [46, 47] so that the discretized form of the kinetic equation reads

$$
\begin{array}{r}
\left(\frac{1}{\Delta t^{k}}+c_{p} \cos \theta \frac{\partial}{\partial r}-\frac{c_{p} \sin \theta}{r} \frac{\partial}{\partial \theta}\right) \Delta f^{k}=R H S^{k}, \\
R H S^{k}=n \delta T^{1-\omega}\left(f^{S^{k}}-f^{k}\right)-c_{p} \cos \theta \frac{\partial f^{k}}{\partial r}+\frac{c_{p} \sin \theta}{r} \frac{\partial f^{k}}{\partial \theta} .
\end{array}
$$

The left hand side of Eq. 85 is the implicit part, where the spacial derivatives are approximated by the first order upwind scheme. The advantage of this discretization is that the solution $\Delta f^{k}$ can be obtained directly, i.e. without calculation of the inverse matrix, just by marching in appropriate direction. The right hand side of Eq. 85) $\left(R H S^{k}\right)$ is the explicit part, where the spacial derivatives are approximated by the second order TVDlike scheme. Minute details concerning the numerical scheme are provided in Ref. [48. The calculations were carried out for three pairs of temperature $T_{s} / T_{0}$ and density $n_{s} / n_{0}$ ratios and for the same values of rarefaction parameter and evaporation-condensation coefficient previously considered when the linear approach was implemented. The grid parameters were chosen to ensure an accuracy of $0.1 \%$ for the moments of the distribution function. While 
the values of the parameters $N_{c}$ and $N_{\theta}$ were fixed at 12 and 400, respectively, the parameter $N_{r}$ varied according to the value of the maximum radial coordinate, $r_{\text {max }}$, so that the increment $\Delta r_{i} \sim 10^{-2}\left(i=0, \ldots, N_{r}\right)$. For the three pairs of $T_{s} / T_{0}$ and $n_{s} / n_{0}$ considered in the calculations, $r_{\max }$ was chosen so that the dimensional quantity $r_{\max }^{\prime}=r_{\max } \delta \ell_{0}$ varied from $10 \ell_{0}$ to $100 \ell_{0}$ as $\delta$ varied from 0.01 to 10 .

It is worth mentioning that the numerical method used to solve the nonlinear kinetic equation did not require a special treatment to deal with the problem of the discontinuity of the perturbation function which appeared when the DVM was employed to solve the linearized kinetic equation. To show the behavior of the normalized distribution function $\hat{f}$ on the angle $\theta$ and fixed $r$ and $c_{p}$ some figures are provided as online supplementary material for small values of $\delta$.

\section{Results and discussion}

We present the results in the following manner. First, the results obtained numerically from the linearized S-model kinetic equation and the analytical solution of the linearized Navier-Stokes equations subject to the pressure and temperature jumps boundary conditions at the interface are presented in order to show the limits of the applicability of this analytical solution in terms of the rarefaction parameter. The cases of complete and non-complete evaporation-condensation are considered. Then, the linearized and non-linear numerical solutions of the S-model kinetic equation are compared to show the limits of the linearized approach application.

\subsection{Linearized approach}

A comparison between the results of the present work, calculated using the discrete velocity method, and those provided in Ref. [26], obtained via the integral-moment method, is presented in Tables 1 and 2 for complete evaporation-condensation at the liquid-vapor interface $(\sigma=1)$ and in a wide range of the gas rarefaction. From these tables one can see a good agreement between the results for the bulk velocities, $u^{(n)}$ and $u^{(T)}$, and heat fluxes, $q^{(n)}$ and $q^{(T)}$, at the interface. The difference between the results is less than $2 \%$ for $u^{(n)}, u^{(T)}$ and $q^{(T)}$, and less than $10 \%$ for $q^{(n)}$.

The results for the mass flow rate and heat flux from the sphere were also compared to the results presented in Ref. [24, which were obtained from the solution of the linearized Boltzmann equation for hard-spheres molecules and complete evaporation-condensation at the interface. The comparison is shown in Tables 3 and 4 . For convenience, the results are presented in terms of the temperature $X_{T}$ and pressure $X_{P}=X_{n}+X_{T}$ driving forces as 
it was done in Ref. [24. Moreover, the results are given as functions of the parameter $k=(\sqrt{\pi} / 2) K n$ introduced in Ref. [24], which is proportional to the Knudsen number $K n$ and can be defined either in terms of the gas viscosity or heat conductivity. As one can see from these tables, there is a good agreement between the present results obtained via the numerical solution of the linearized S-model kinetic equation and the data reported in Ref. [24]. The difference between the results is smaller than $2 \%$ in the whole range of the parameter $k$. The figures showing the comparison between the present results and those from both Refs. [24, 26] for the mass flow rate and heat flux is available as online suplementary material. The present results are in a better agreement with those provided in Ref. 24 than those obtained by the authors of Ref. [26]. It is worth noting that the integral-moment method employed in Ref. [26] consists on obtaining a set of integral equations for the moments of the distribution function and its advantage is that only the physical space must be discretized. Nevertheless, this method requires much more computational memory and CPU time than that required by the discrete velocity method. Moreover, even with the computational infrastructure available nowadays, the numerical solution of the Boltzmann equation is still a hard task. Therefore, the implementation of the model kinetic equation allows us to solve the problem with good accuracy and moderate computational effort. Since the results of the present work and those provided in Ref. [26] were obtained by the solution of the same kinetic equation, one can conclude that the better agreement with the results from the Boltzmann equation is due to the numerical scheme used in this work.

Figures 1.3 show the profiles of the bulk velocities, $u^{(n)}$ and $u^{(T)}$, and heat fluxes, $q^{(n)}$ and $q^{(T)}$, as functions of the dimensionless radial coordinate $r$, for the rarefaction parameter, $\delta$, equal to $0.1,1$ and 10, respectively. For each value of rarefaction parameter, four values of the evaporation-condensation coefficient are considered, $\sigma=0.1,0.4,0.6$ and 1 . In these figures, the dimensionless radial distance $r=10$ corresponds to a dimensional radial distance $r^{\prime}=10 R_{0}=10 \delta \ell_{0}$. According to these figures, the larger the rarefaction parameter the larger the bulk velocities in the vicinity of the sphere due to the driving forces $X_{n}$ and $X_{T}$. The opposite behavior occurs for the heat fluxes, i.e. the larger the rarefaction parameter the smaller the magnitude of the heat fluxes in the vicinity of the spherical droplet. Note that $q^{(n)}$ is negative, while $q^{(T)}$ is positive. These figures also show us that the influence of the evaporation-condensation coefficient, $\sigma$, on the bulk velocities $u^{(n)}$ and $u^{(T)}$, and heat flux $q^{(n)}$ is significant, while the influence on the heat flux $q^{(T)}$ can be neglected whatever the value of the gas rarefaction. In fact, the heat flux $q^{(T)}$ depends only on the temperature difference between the droplet surface and the gas in equilibrium far from the droplet. The same 
qualitative behavior was found in Ref. [28], in which the problem of noncomplete evaporation-condensation between two parallel condensed phases was simulated. For instance, in case of $\delta=10$, the numerical results obtained from the kinetic equation at the interface, given in Tables 5 and 6, show a difference of about $93 \%$ for the bulk velocities $u^{(n)}$ and $u^{(T)}$, and heat flux $q^{(n)}$, when the evaporation-condensation coefficient varies from 1 to 0.1 . On the other hand, the difference for the heat flux $q^{(T)}$ is less than $20 \%$. These results show us that the evaporation-condensation on the spherical droplet depends not only on the gas rarefaction degree, but depends strongly on the evaporation-condensation coefficient at the interface.

The comparison between the solution of the linearized kinetic equation and the solution obtained from the Navier-Stokes equations given in (72) and (71) is shown in Figure 4 for $\delta=10$ and complete evaporation-condensation at the interface. According to this figure, there is a good agreement between both results. Thus, in order to do just an estimation of the evaporation parameters from the spherical droplet, as well as of the flow fields around it, the analytical solution obtained from the linearized Navier-Stokes equations subjected to the temperature and pressure jump boundary conditions can be used instead of the solution of the linearized kinetic equation. Tables 5 and 6 show the comparison in case of complete and non-complete evaporationcondensation at the interface when $\delta=10$. Note that, just for comparison, the results obtained in the limit of $\delta \rightarrow \infty$, given in Eqs. (72) and (74), were also included in the tables. Thus, as one can see from Tables 5 and 6 , there is a good agreement between the resuls, and such an agreement will be improved with the increase of the rarefaction parameter. Moreover, a better agreement depends on the use of more precise values of the pressure and temperature jump coefficients. In the present work, the temperature and pressure jump coefficients at the interface provided in Ref. 20] were used, but these coefficients were calculated via the solution of the BGK-model kinetic equation [49] and the variational method.

The comparison of the widely used Schrage formula $(76)$ with the analytical solution obtained from the linearized Navier-Stokes equations with the temperature and pressure jump boundary conditions $(75)$ at the interface and the numerical solution of the linearized kinetic equation for $\delta=10$ are presented in Table 7. From this table it is clear that the analytical solution derived from the Navier-Stokes equations shows a better agreement with the numerical solution of the kinetic equation compared to the Schrage expression. As it was mentioned previously, the discrepancy between the solution of the kinetic equation for $\delta=10$ and the analytical solution obtained from the Navier-Stokes equations could be explained by the accuracy of the coefficients involved in the temperature and pressure jump conditions. 


\subsection{Comparison between linearized and non-linear S-model solution}

The comparison of the results obtained from the linearized approach and that from the solution of the non-linear kinetic equation are presented in Tables 8,10 for different values of the rarefaction parameter and evaporation coefficients. This comparison can only be done when the driving forces are specified to calculate the macroscopic parameters from the numerical data given by the linearized approach. Three pairs of the temperature ratios, $T_{s} / T_{0}$, and corresponding number density ratios, $n_{s} / n_{0}$, are chosen for this comparison. According to Table 8, for very small deviation from the equilibrium values, $T_{s} / T_{0}=1.01$ and $n_{s} / n_{0}=1.01$, both approaches provide very similar results, with a maximal deviation of less than $1 \%$ for the velocity and around $2.5 \%$ for the heat flux. However, in practice, following the ClausiusClayperon equation [33], the small deviation in temperature leads to a deviation in pressure and, therefore, in the number density, ten times larger. Thus, the results for the ratios $T_{s} / T_{0}=1.01$ and $n_{s} / n_{0}=1.1$ are presented in Table 9 for analysis. As it is clear from Table 9 , in this case the difference between the bulk velocity and heat flux calculated by linearized and non-linear approaches is larger, of the order of $4 \%$ for the velocity and $6 \%$ for the heat flux. Finally, the larger temperature deviation is considered, corresponding to $5 \%$ from its equilibrium value. In this case, according to the Clapeyron data [33], the density deviation is ten times larger, i.e. $n_{s} / n_{0}=1.5$. As it is clear from Table 10, a large discrepancy between linearized and non-linear results are observed, of the order of $25 \%$ for both quantities, the macroscopic velocity and the heat flux. It is to note that for all considered values of the evaporation coefficient the difference between linearized and non-linear results is maximum for the complete evaporation, $\sigma=1$. Thus, according to the results, the linearized approach could be applied only when the temperature deviation is less than $5 \%$.

\section{Conclusion}

The steady weak evaporation from or condensation onto a spherical condensed phase in its vapor was studied numerically by using the linearized and non-linear S-model kinetic equations. When using the linearized approach, the obtained results have been found in good agreement with those obtained from the linearized Boltzmann equation (maximum difference of $2 \%$ ) and with those obtained from the solution of the S-model via the integral-moment method (maximum difference of 10\%) in the case of complete evaporationcondensation at the interface. In the free molecular flow regime, the analytical solution was obtained in case of complete and non-complete evaporationcondensation at the droplet surface. In the hydrodynamic regime, the lin- 
earized Navier-Stokes equations subjected to the temperature and pressure jump boundary conditions were solved analytically and the obtained results are in good agreement to those calculated from the numerical solution of the the kinetic equation in the case of $\delta=10$. The improvement of this agreement could be probably reached when using the more precise values for the pressure and temperature jumps at the interface. The widely used Schrage expression for the mass flow rate was compared to that derived from the analytical solution of the Navier-Stokes equations and also with the numerical results obtained from the linearized kinetic equation when $\sigma=0.1,0.5$ and 1 . It was found that the analytical solution of the Navier-Stokes equations with the jump conditions provides the values of the mass flow rate closer to the values obtained from the numerical solution of the S-model kinetic equation than tha values predicted by the Schrage expression.

The non-linear S-model kinetic equation was solved by the implicit TVD type numerical method, which does not need any special treatment for the discontinuity of the molecular velocity distribution function at the interface. The results obtained from the linearized and non-linear approaches were compared for several typical ratios of temperatures and the number densities. It was found that the linearized approach could be applied when the temperature difference between the droplet surface and the gas in equilibrium far from the doplet does not exceed $5 \%$. If this difference is larger than $5 \%$, the non-linear approach must to be applied.

The results obtained in the present work show us a strong dependence of the flow fields on the evaporation-condensation coefficient so that one cannot always rely on the results for complete evaporation-condensation at the liquid-vapor interface. In fact, a better understanding of the evaporationcondensation at the interface of a spherical droplet relies on a better description of the phase transition at the interface of the droplet and further investigation on this research topic must be encouraged. Moreover, since the results available in the literature for non-complete evaporation-condensation at the interface are still scarce, the results obtained in the present work represent a significant contribution towards a better understanding of the evaporation-condensation phenomena at the interface of spherical droplets.

\section{Acknowledgments}

Vinicius Braga Leite and Denize Kalempa acknowledge the Fundação de Amparo à Pesquisa do Estado de São Paulo (FAPESP), grants 2016/20716-9, 2016/09118-2 and 2015/20650-5, for the support of the research. 


\section{Supplementary material}

Supplementary material is available.

Figures 1 and 2 show the comparison between our results and those provided in Refs. [24, 26] for the mass and heat flux from the sphere as functions of the rarefaction parameter and in case of complete evaporationcondensation at the interface.

Figure 3 shows the profiles of the perturbation functions $h^{(n)}$ and $h^{(T)}$ on the angle $\theta$ at $r=1.975$ and molecular speed $c_{p} \approx 2.3$ obtained from the linearized approach for small values of the rarefaction parameter and complete evaporation-condensation at the interface. The solutions obtained from the DVM with and without the split method are presented for comparison.

Figure 4 shows the profile of the normalized distribution function $\hat{f}$ on the angle $\theta$ at $r=1.975$ and $c_{p} \approx 2.3$ obtained from the linearized and nonlinear approaches for small values of the rarefaction parameter and complete evaporation-condensation at the interface. 


\section{Appendix A. Split method to solve the linearized kinetic equation}

The differential equations given by (54) for each thermodynamic force and subject to the corresponding boundary condition, given by (55) and (56), are solved analytically via the method of characteristics and the solutions are presented as follows

$$
\begin{aligned}
& h_{0}^{(n)}\left(r, \theta, c_{p}\right)=\left\{\begin{array}{l}
\sigma \exp \left(-\frac{\delta S}{c_{p}}\right), \quad 0 \leq \theta \leq \theta_{0}, \\
0, \quad \theta_{0}<\theta \leq \pi,
\end{array}\right. \\
& h_{0}^{(T)}\left(r, \theta, c_{p}\right)=\left\{\begin{array}{l}
\left(c_{p}^{2}-2+\frac{\sigma}{2}\right) \exp \left(-\frac{\delta S}{c_{p}}\right), \quad 0 \leq \theta \leq \theta_{0}, \\
0, \quad \theta_{0}<\theta \leq \pi,
\end{array}\right.
\end{aligned}
$$

where

$$
S=r \cos \theta-\sqrt{1-r^{2} \sin ^{2} \theta}
$$

is the distance between a point in the gas flow domain with Cartesian coordinates $(x, y, z)$ and a point on the spherical surface with Cartesian coordinates $\left(x_{0}, y_{0}, z_{0}\right)$ directed towards -c.

The angle $\theta_{0}$ is given by

$$
\theta_{0}=\arcsin \left(\frac{1}{r}\right)
$$

Thus, from 21)-24, the moments of the functions $h_{0}^{(i)}$ are written as

$$
\begin{aligned}
\nu_{0}^{(n)}(r) & =\frac{2 \sigma}{\sqrt{\pi}} \int_{0}^{\theta_{0}} \sin \theta I_{2}(\delta S) \mathrm{d} \theta, \\
\tau_{0}^{(n)}(r) & =\frac{4 \sigma}{3 \sqrt{\pi}} \int_{0}^{\theta_{0}} \sin \theta\left[I_{4}(\delta S)-\frac{3}{2} I_{2}(\delta S)\right] \mathrm{d} \theta, \\
u_{0}^{(n)}(r) & =\frac{2 \sigma}{\sqrt{\pi}} \int_{0}^{\theta_{0}} \sin \theta \cos \theta I_{3}(\delta S) \mathrm{d} \theta, \\
q_{0}^{(n)}(r) & =\frac{2 \sigma}{\sqrt{\pi}} \int_{0}^{\theta_{0}} \sin \theta \cos \theta\left[I_{5}(\delta S)-\frac{5}{2} I_{3}(\delta S)\right] \mathrm{d} \theta,
\end{aligned}
$$




$$
\begin{gathered}
\nu_{0}^{(T)}(r)=\frac{2}{\sqrt{\pi}} \int_{0}^{\theta_{0}} \sin \theta\left[I_{4}(\delta S)+\frac{1}{2}(\sigma-4) I_{2}(\delta S)\right] \mathrm{d} \theta \\
\tau_{0}^{(T)}(r)=\frac{4}{3 \sqrt{\pi}} \int_{0}^{\theta_{0}} \sin \theta\left[I_{6}(\delta S)+\frac{1}{2}(\sigma-7) I_{4}(\delta S)-\frac{3}{2}(\sigma-2) I_{2}(\delta S)\right] \mathrm{d} \theta \\
u_{0}^{(T)}(r)=\frac{2}{\sqrt{\pi}} \int_{0}^{\theta_{0}} \sin \theta \cos \theta\left[I_{5}(\delta S)+\frac{1}{2}(\sigma-4) I_{3}(\delta S)\right] \mathrm{d} \theta, \\
q_{0}^{(T)}(r)=\frac{2}{\sqrt{\pi}} \int_{0}^{\theta_{0}} \sin \theta \cos \theta\left[I_{7}(\delta S)+\frac{1}{2}(\sigma-9) I_{5}(\delta S)-\frac{5}{4}(\sigma-4) I_{3}(\delta S)\right] \mathrm{d} \theta
\end{gathered}
$$

The functions $I_{m}$ are defined as

$$
I_{m}(\delta S)=\int_{0}^{\infty} c_{p}^{m} \exp \left(-c_{p}^{2}-\frac{\delta S}{c_{p}}\right) \mathrm{d} c_{p}
$$

and are calculated by using the power series representation given in Ref. [50].

\section{References}

[1] M. M. Awad, A. S. Dalkiliç, and S. Wongwises. A critical review on condensation heat transfer in microchannels and minichannels. J. Nanotechnol. Eng. Med., 51, 2014.

[2] S. G. Kanklikar, S. Colin, Y Peles, S. Garimella, R. F. Pease, and J. J. Brandner. Heat transfer in microchannels - 2012 status and research needs. J. Heat Transfer, 135(0910011), 2013.

[3] A. Bar-Cohen and P. Wang. Thermal management of on-chip hot spot. J. Heat Transfer, 134, 2012.

[4] H. Hertz. Uber die Verdungstung der Flussigkeiten, inbesondere des Quecksilbers, im luftleeren Raume. Ann. Phys. Chem., 17:177-200, 1882. 
[5] M. Knudsen. Die Maximale Verdampfungsgeschwindigkeit des Quecksilbers. Ann. Phys. Chem., 47:697-708, 1915.

[6] Y. P. Pao. Temperature and density jumps in the kinetic theory of gases and vapors. Phys. Fluids, 14(7):1340-1346, 1971.

[7] Y. P. Pao. Application of kinetic theory to the problem of evaporation and condensation. Phys. Fluids, 14(2):306-312, 1971.

[8] L. H. Thomas, T. S. Chang, and S. E. Siewert. Reverse temperature gradient in the kinetic theory of evaporation. Phys. Rev. Lett., 33:680$682,1974$.

[9] D. A. Labuntsov. Analysis of the evaporation and condensation processes. High Temp., 5:579-585, 1967.

[10] D. A. Labuntsov and A. P. Kryukov. Analysis of intensive evaporation and condensation. Int. J. Heat Mass Transfer, 22:989-1002, 1979.

[11] P. N. Shankar and M. D. Deshpande. On the temperature distribution in liquid-vapor phase change between plane liquid surfaces. Phys. Fluids A, 2:1030-1038, 1990 .

[12] C. A. Ward and G. Fang. Expression for predicting liquid evaporation flux: Statistical rate theory approach. Phys. Rev. E, 59:429-440, 1999.

[13] C. A. Ward and D. Stanga. Interfacial conditions during evaporation or condensation of water. Phys. Rev. E, 64:051509, 2001.

[14] V. K. Badam, V. Kumar, F. Durst, and K. Danov. Experimental and theoretical investigations on interfacial temerature jumps during evaporation. Experimental Thermal and Fluid Science, 32:276-292, 2007.

[15] E. Ya Gatapova, I. Graur, F. Sharipov, and O. A. Kabov. The temperature and pressure jumps at the vapor-liquid interface: Application to a two-phase cooling system. Int. J. Heat Mass Transfer, 83:235-243, 2015 .

[16] E. Ya Gatapova, I. Graur, O. A. Kabov, V. A. Aniskin, M. A. Filipenko, and F. Sharipov. The temperature jump at water-air interface during evaporation. Int. J. Heat Mass Transfer, 2017.

[17] M. A. Kazemi, D. S. Nobes, and J. A. W. Elliott. Experimental and numerical study of the evaporation of water at low pressures. Langmuir, 33(18):4578-4591, 2017. 
[18] P. Jafari, A. Masoudi, P. Irajizad, M. Nazari, V. Kashyap, B. Eslami, and H. Ghasemi. Evaporation mass flux: a predictive model and experiments. Langmuir, 34:11676-11684, 2018.

[19] A. P. Kryukov and V. Yu ans Sazhin S. S. Levashov. Evaporation of diesel fuel droplets: kinetic versus hydrodynamic models. Int. J. Heat Mass Transfer, 47:2541, 2004.

[20] J. W. Cipolla Jr, H. Lang, and S. K. Loyalka. Kinetic theory of condensation and evaporation. II. J. Chem. Phys., 61:69-77, 1974.

[21] Y. Sone and Y. Onishi. Kinetic theory of evaporation and condensation - Hydrodynamic equation and slip boundary condition. J. Phys. Soc. Japan, 44:1981-1994, 1978.

[22] Y. Sone and Y. Onishi. Kinetic theory of evaporation and condensation. J. Phys. Soc. Japan, 35:1773-1776, 1973.

[23] Y. Sone, T. Ohwada, and K. Aoki. Evaporation and condensation on a plane condensed phase: numerical analysis of the linearized Boltzmann equation for hard-sphere molecules. Phys. Fluids A, 1:1398-1405, 1989.

[24] S. Takata, Y. Sone, D. Lhuillier, and M. Wakabayashi. Evaporation from or condensation onto a sphere: numerical analysis of the Boltzmann equation for hard-sphere molecules. Computers Math. Applic., 35(1/2):193-214, 1998.

[25] J. B. Young. The condensation and evaporation of liquid droplets in a pure vapour at arbitrary Knudsen number. Int. J. Heat Mass Transfer, 34(7):1649-1661, 1990.

[26] V. G. Chernyak and A. Ye Margilevskiy. The kinetic theory of heat and mass transfer from a spherical particle in a rarefied gas. Int. J. Heat Mass Transfer, 32(11):2127-2134, 1989.

[27] K Aoki and N Masukawa. Gas flows caused by evaporation and condensation on two parallel condensed phases and the negative temperature gradient: numerical analysis by using a nonlinear kinetic equation. Phys. Fluids, 6(3):1379-1395, 1994.

[28] A. Polikarpov and I. Graur. Heat and mass transfer in a rarefied gas confined between its two parallel condensed phases. Int. J. Heat Mass Transfer, 124:967-979, 2018. 
[29] E M Shakhov. Approximation kinetic equations in rarefied gas theory. Fluid Dynamics, 3(1):112-115, 1968.

[30] Y. Sone. Thermal creep in rarefied gas. J. Phys. Soc. Japan, 21:1836$1837,1966$.

[31] Y. Sone and S. Takata. Discontinuity of the velocity distribution function in a rarefied gas around a convex body and the $\mathrm{S}$ layer at the bottom of the Knudsen layer. Trans. Theory Stat. Phys., 21(4-6):501-530, 1992.

[32] M. T. Ho and I. Graur. Heat transfer through rarefied gas confined between two concentric spheres. Int. J. Heat Mass Transfer, 90:58-71, 2015 .

[33] L. D. Landau and E. M. Lifshitz. Fluid Mechanics. Pergamon, New York, 1989.

[34] N. A. Fuchs. Evaporation and Droplet Growth in Gaseous Media. Pergamon Press, London, 1959.

[35] G. M. Hidy and J. R. Brock. The Dynamics of Aerocolloidal Systems. Pergamon Press, London, 1970.

[36] M. N. Kogan. Rarefied Gas Dynamics. Plenum, New York, 1969.

[37] J. H. Ferziger and H. G. Kaper. Mathematical Theory of Transport Processes in Gases. North-Holland Publishing Company, Amsterdam, 1972.

[38] S. R. De Groot and P. Mazur. Non-Equilibrium Thermodynamics. Dover Publications, Inc., New York, 1984.

[39] F. Sharipov. Rarefied Gas Dynamics. Fundamentals for Research and Practice. Wiley-VCH, Berlin, 2016.

[40] S. Naris and D. Valougeorgis. The driven cavity flow over the whole range of the Knudsen number. Phys. Fluids, 17(9):097106, 2005.

[41] Y Sone. Molecular Gas Dynamics. Theory, Techniques and Applications. Birkhäuser, Boston, 2007.

[42] V. I. Krylov. Approximate Calculation of Integrals. Dover Publication Inc., Mineola, 2005.

[43] S. Fujikawa, T. Yano, and M. Watanabe. Vapor-liquid interface, bubbles and droplets. Springer, 2011. 
[44] J. W. Cipolla Jr and T. F. Morse. Kinetic description of cylindrical heat conduction in a polyatomic gas. Phys. Fluids, 11(6):1292-1300, 1968.

[45] A. Schrage. A Theoretical Study of Interphase Mass Transfer. Columbia University Press, New York, 1953.

[46] J. Y. Yang and J. C. Huang. Rarefied flow computations using nonlinear model Boltzmann equations. J. Comput Phys, 120(2):323-339, 1995.

[47] V. A. Titarev and E. M. Shakhov. Numerical study of unsteady evaporation and heat transfer from a spherical surface. Fluid Dynamics, 40(1):159-168, 2005.

[48] I. Graur, M. T. Ho, and M. Wuest. Simulation of the transient heat transfer between two coaxial cylinders. J. Vac. Sci. Technol. A, 31(6), 2013.

[49] P. L. Bhatnagar, E. P. Gross, and M. A. Krook. A model for collision processes in gases. Phys. Rev., 94:511-525, 1954.

[50] M. Abramowitz and I. A. Stegun. Handbook of Mathematical Functions with formulas, graphs and mathematical tables. Dover Publications Inc., New York, 9 edition, 1972. 
Table 1: Bulk velocities, $u^{(n)}$ and $u^{(T)}$, at the liquid-vapor interface $(r=1)$ in case of complete evaporation-condensation $(\sigma=1)$. Comparison with the numerical data of Ref. [26.

\begin{tabular}{ccc|cc}
\hline \multicolumn{3}{c|}{$u^{(n)}$} & \multicolumn{2}{c}{$u^{(\mathrm{T})}$} \\
\hline$\delta$ & present work & Ref. [26] & present work & Ref. [26] \\
\hline $0^{\mathrm{a}}$ & 0.282 & - & 0.141 & - \\
0.01 & 0.283 & 0.284 & 0.141 & 0.142 \\
0.1 & 0.289 & 0.293 & 0.147 & 0.148 \\
0.5 & 0.312 & 0.317 & 0.172 & 0.176 \\
1 & 0.330 & 0.336 & 0.203 & 0.206 \\
5 & 0.401 & 0.402 & 0.333 & 0.338 \\
7 & 0.415 & 0.415 & 0.361 & 0.366 \\
10 & 0.424 & 0.426 & 0.385 & 0.390 \\
$\infty^{\mathrm{b}}$ & 0.470 & - & 0.470 & - \\
\hline
\end{tabular}

a Eq. 49, free molecular regime.

b Eq. 72, hydrodynamic regime.

Table 2: Heat fluxes, $q^{(n)}$ and $q^{(\mathrm{T})}$, at the liquid-vapor interface $(r=1)$ in case of complete evaporation-condensation $(\sigma=1)$. Comparison with the numerical data of Ref. [26].

\begin{tabular}{ccc|cc}
\hline \multicolumn{3}{c|}{$-q^{(n)}$} & \multicolumn{2}{c}{$q^{(\mathrm{T})}$} \\
\hline$\delta$ & present work & Ref. [26] & present work & Ref. [26] \\
\hline $0^{\mathrm{a}}$ & 0.141 & - & 0.494 & - \\
0.01 & 0.141 & 0.142 & 0.492 & 0.495 \\
0.1 & 0.142 & 0.144 & 0.484 & 0.491 \\
0.5 & 0.139 & 0.141 & 0.443 & 0.454 \\
1 & 0.131 & 0.130 & 0.399 & 0.405 \\
5 & 0.0673 & 0.0635 & 0.204 & 0.200 \\
7 & 0.0548 & 0.0510 & 0.161 & 0.159 \\
10 & 0.0394 & 0.0360 & 0.123 & 0.121 \\
$\infty^{\mathrm{b}}$ & 0.000 & - & 0.000 & - \\
\hline
\end{tabular}

${ }^{\text {a }}$ Eq. (50), free molecular regime.

b Eq. 71, hydrodynamic regime. 
Table 3: Mass flow rates from the sphere in case of complete evaporation-condensation $(\sigma=1)$. Comparison with the numerical data of Ref. 24]. Here, the parameter $k$ is related to the rarefaction parameter $\delta$ as $k=1 /\left(\gamma_{1} \delta\right)$ with $\gamma_{1}=1.270042$.

\begin{tabular}{ccc|cc}
\hline & \multicolumn{2}{c|}{$r^{2} u^{(P)}$} & \multicolumn{2}{c}{$-r^{2} u^{(T)}$} \\
\cline { 2 - 5 }$k$ & present work & Ref. [24] & present work & Ref. [24] \\
\hline 0.1 & 0.419 & 0.417 & 0.0449 & 0.0441 \\
0.2 & 0.389 & 0.385 & 0.0751 & 0.0740 \\
0.4 & 0.358 & 0.351 & 0.108 & 0.106 \\
0.6 & 0.340 & 0.334 & 0.121 & 0.120 \\
1 & 0.323 & 0.317 & 0.133 & 0.132 \\
2 & 0.305 & 0.301 & 0.141 & 0.139 \\
4 & 0.294 & 0.292 & 0.142 & 0.141 \\
6 & 0.291 & 0.289 & 0.142 & 0.141 \\
10 & 0.288 & 0.286 & 0.142 & 0.141 \\
20 & 0.285 & 0.284 & 0.142 & 0.141 \\
\hline
\end{tabular}

Table 4: Heat fluxes from the sphere in case of complete evaporation-condensation $(\sigma=1)$. Comparison with the numerical data of Ref. 24]. Here, the parameter $k$ is related to the rarefaction parameter $\delta$ as $k=3 /\left(2 \gamma_{2} \delta\right)$ with $\gamma_{2}=1.92284$.

\begin{tabular}{ccc|cc}
\hline & \multicolumn{2}{c|}{$-r^{2} q^{(P)}$} & \multicolumn{2}{c}{$r^{2} q^{(T)}$} \\
\cline { 2 - 5 }$k$ & present work & Ref. [24] & present work & Ref. [24] \\
\hline 0.1 & 0.0449 & 0.0441 & 0.195 & 0.193 \\
0.2 & 0.0751 & 0.0741 & 0.312 & 0.314 \\
0.4 & 0.108 & 0.106 & 0.436 & 0.441 \\
0.6 & 0.122 & 0.120 & 0.496 & 0.503 \\
1 & 0.133 & 0.132 & 0.552 & 0.558 \\
2 & 0.141 & 0.139 & 0.600 & 0.600 \\
4 & 0.142 & 0.141 & 0.619 & 0.619 \\
6 & 0.142 & 0.141 & 0.625 & 0.625 \\
10 & 0.142 & 0.141 & 0.629 & 0.629 \\
20 & 0.142 & 0.141 & 0.632 & 0.632 \\
\hline
\end{tabular}



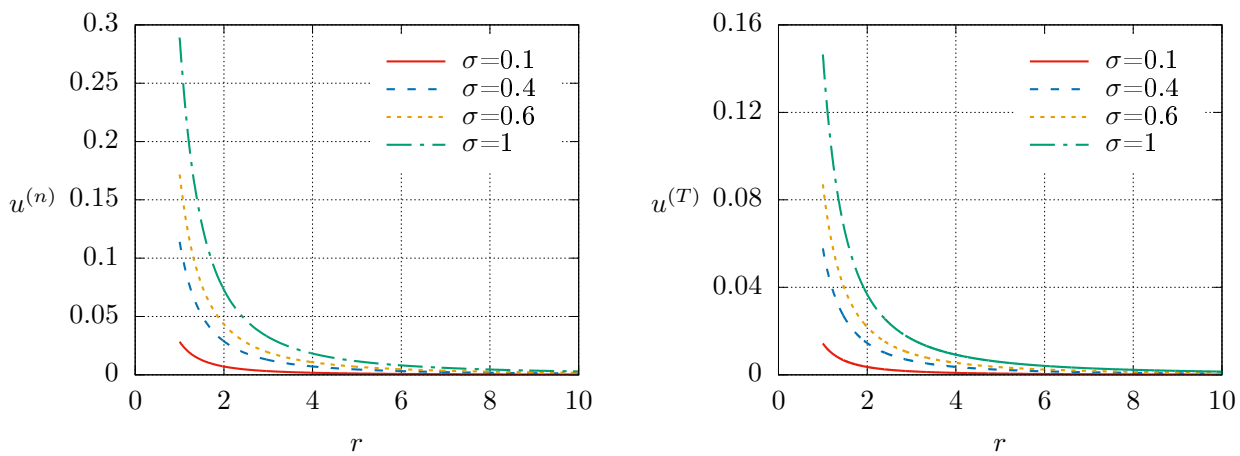

(a) Bulk velocity
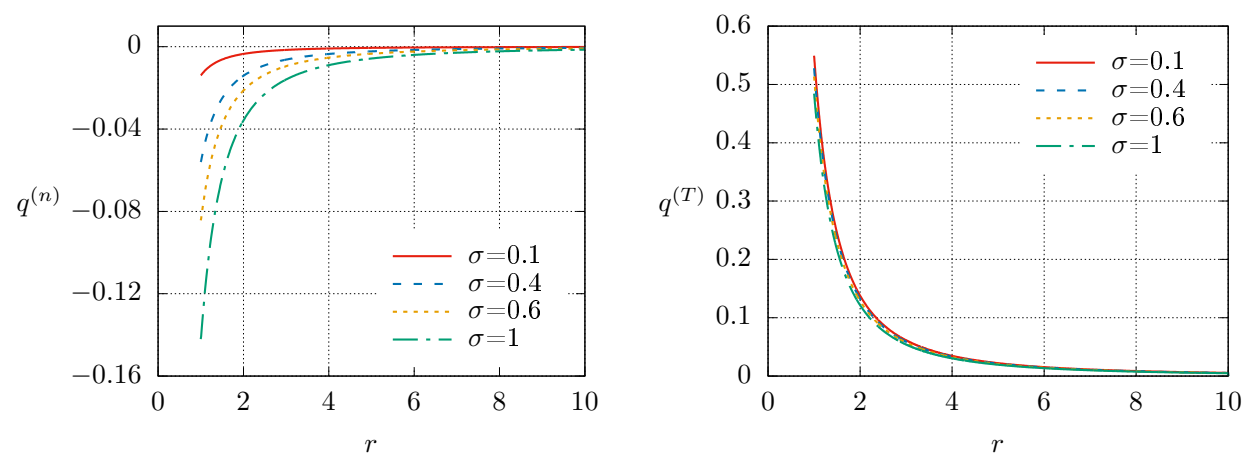

(b) Heat flux

Figure 1: Rarefaction parameter $\delta=0.1$ 

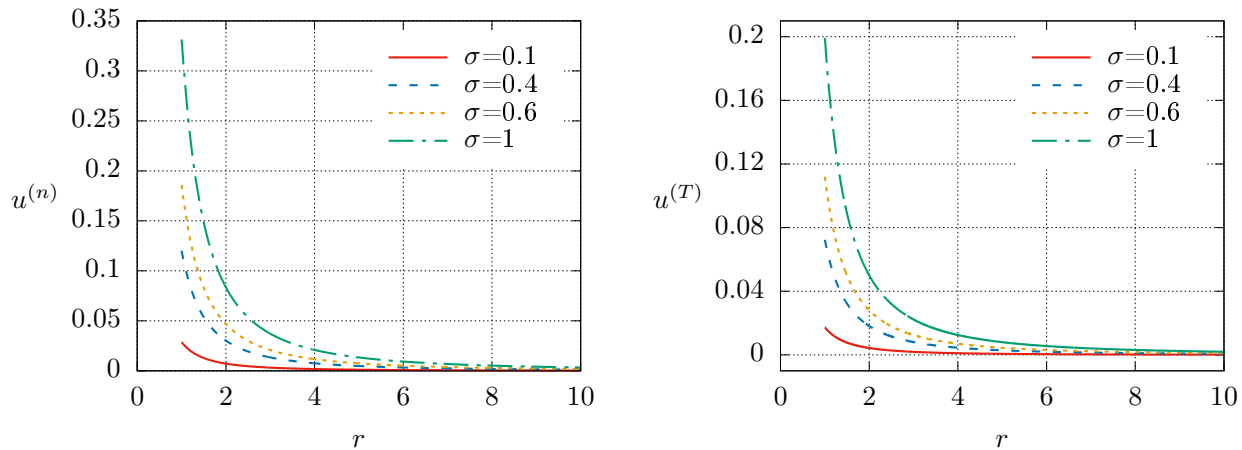

(a) Bulk velocity
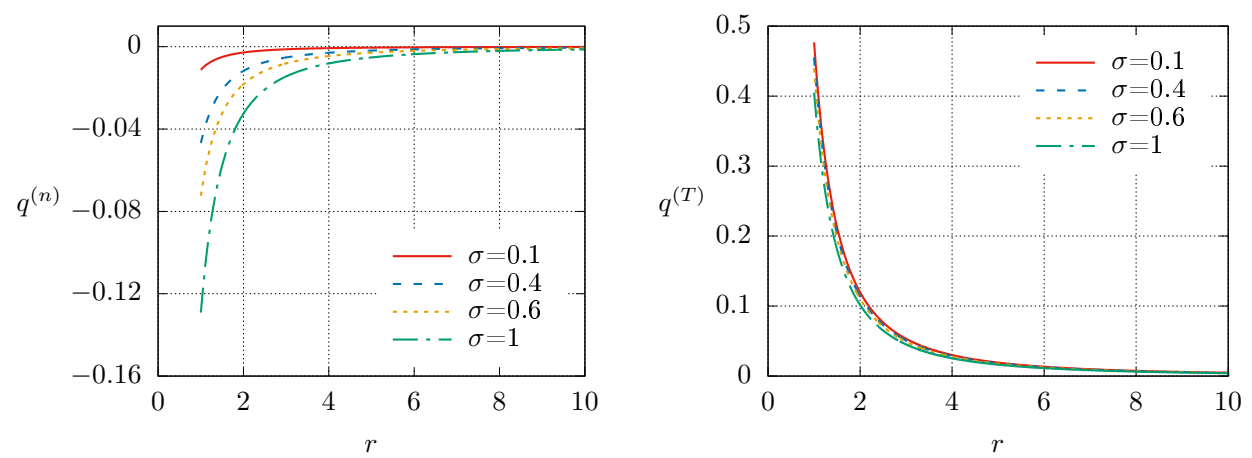

(b) Heat flux

Figure 2: Rarefaction parameter $\delta=1$ 

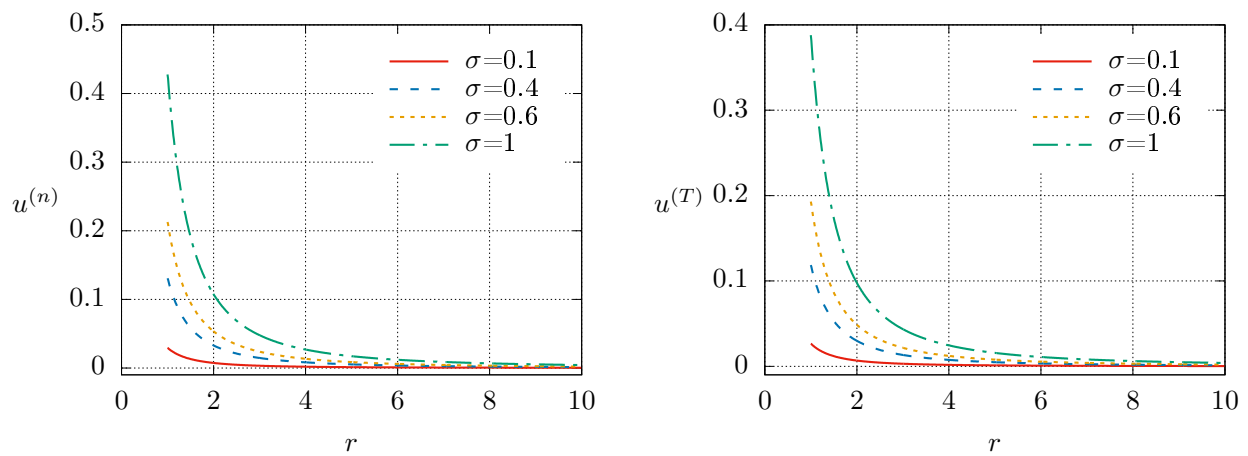

(a) Bulk velocity
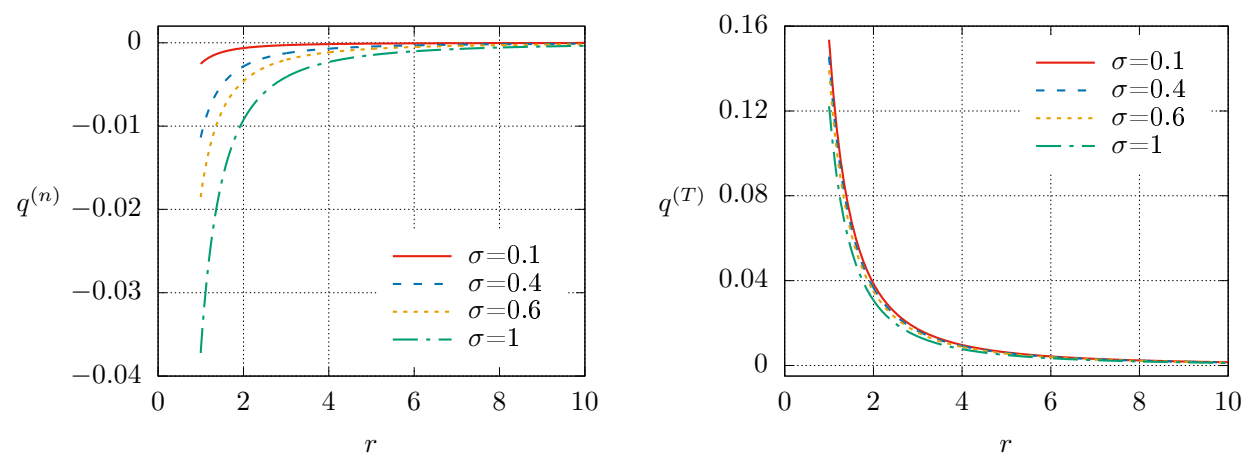

(b) Heat flux

Figure 3: Rarefaction parameter $\delta=10$ 

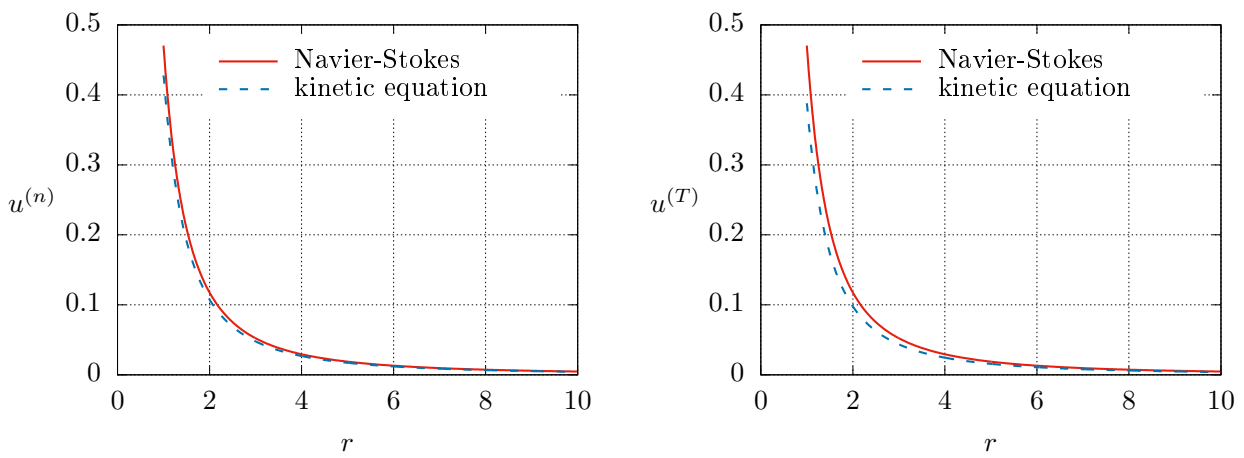

(a) Bulk velocity
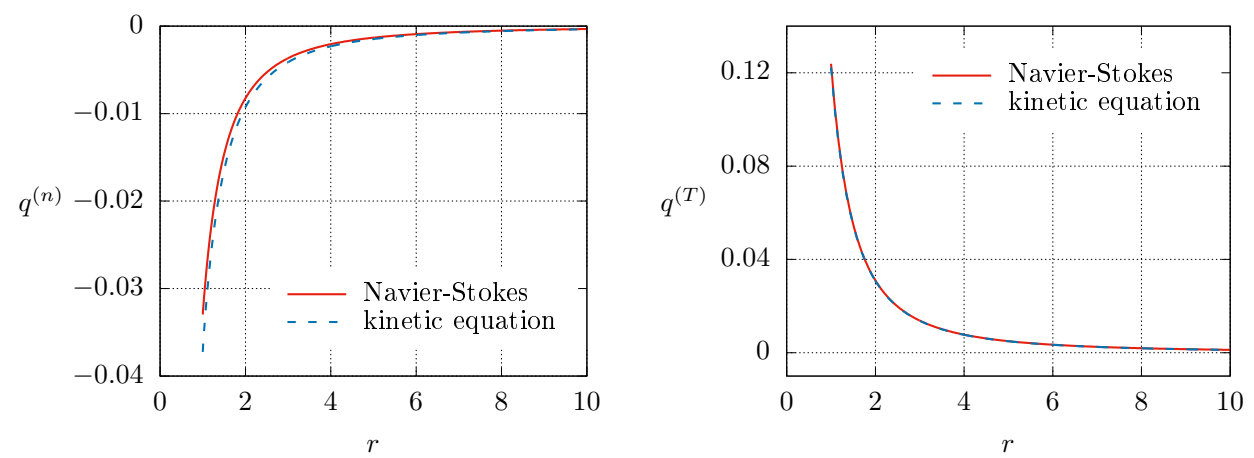

(b) Heat flux

Figure 4: Comparison: Navier-Stokes and kinetic equation, $\delta=10$. 
Table 5: Comparison between the results at the interface liquid-vapor due to the driven force $X_{n}$ : kinetic equation versus Navier-Stokes when $\delta=10$.

\begin{tabular}{cccc|ccc}
\hline & \multicolumn{3}{c|}{$u^{(n)}$} & \multicolumn{3}{c}{$-q^{(n)}$} \\
\cline { 2 - 7 }$\sigma$ & Eq. $(\sqrt{67)}$ & Eq. $(\sqrt{72})$ & kin. eq. & Eq. $(\sqrt{71})$ & Eq. $(74)$ & kin. eq. \\
\hline 0.1 & 0.0294 & 0.0294 & 0.0292 & 0.00206 & 0.00206 & 0.00254 \\
0.4 & 0.135 & 0.134 & 0.131 & 0.0094 & 0.0094 & 0.0114 \\
0.6 & 0.224 & 0.223 & 0.213 & 0.0157 & 0.0156 & 0.0185 \\
1. & 0.477 & 0.470 & 0.426 & 0.0334 & 0.0330 & 0.0394 \\
\hline
\end{tabular}

Table 6: Comparison between the results at the interface liquid-vapor due to the driven force $X_{T}$ : kinetic equation versus Navier-Stokes when $\delta=10$.

\begin{tabular}{cccc|ccc}
\hline & \multicolumn{3}{c|}{$u^{(T)}$} & \multicolumn{3}{c}{$q^{(T)}$} \\
\cline { 2 - 7 }$\sigma$ & Eq. $(\sqrt{67})$ & Eq. $(\sqrt{72})$ & kin. eq. & Eq. $(\overline{71})$ & Eq. $(74)$ & kin. eq. \\
\hline 0.1 & 0.0273 & 0.0294 & 0.0265 & 0.155 & 0.155 & 0.153 \\
0.4 & 0.125 & 0.134 & 0.118 & 0.148 & 0.147 & 0.146 \\
0.6 & 0.209 & 0.223 & 0.193 & 0.142 & 0.141 & 0.139 \\
1. & 0.444 & 0.470 & 0.385 & 0.126 & 0.124 & 0.123 \\
\hline
\end{tabular}

Table 7: Comparison between the results obtained for the mass flow rate in the hydrodydnamic regime with those provided by the Schrage relation

\begin{tabular}{cccccc}
\hline \multirow{6}{*}{$\sigma=0.1$} & $T / T_{0}$ & $n / n_{0}$ & Eq. $(76)$ & Eq. $(75)$ & kin. eq. $(\delta=10)$ \\
\cline { 2 - 6 } & 1.01 & 1.01 & $5.877 \times 10^{-4}$ & $4.454 \times 10^{-4}$ & $5.573 \times 10^{-4}$ \\
& 1.01 & 1.1 & $3.232 \times 10^{-3}$ & $3.118 \times 10^{-3}$ & $3.185 \times 10^{-3}$ \\
& 1.05 & 1.5 & $1.616 \times 10^{-2}$ & $1.559 \times 10^{-2}$ & $1.593 \times 10^{-2}$ \\
\hline$\sigma=0.5$ & 1.01 & 1.01 & $3.527 \times 10^{-3}$ & $2.821 \times 10^{-3}$ & $3.242 \times 10^{-3}$ \\
& 1.01 & 1.1 & $1.940 \times 10^{-2}$ & $1.975 \times 10^{-2}$ & $1.858 \times 10^{-2}$ \\
& 1.05 & 1.5 & $9.700 \times 10^{-2}$ & $9.873 \times 10^{-2}$ & $9.270 \times 10^{-2}$ \\
\hline$\sigma=1$ & 1.01 & 1.01 & $9.410 \times 10^{-3}$ & $8.463 \times 10^{-3}$ & $8.157 \times 10^{-3}$ \\
& 1.01 & 1.1 & $5.175 \times 10^{-2}$ & $5.924 \times 10^{-2}$ & $4.664 \times 10^{-2}$ \\
& 1.05 & 1.5 & $2.590 \times 10^{-1}$ & $2.960 \times 10^{-1}$ & $2.332 \times 10^{-1}$ \\
\hline
\end{tabular}


Table 8: Comparison between linear and non-linear results for the bulk velocity and heat flux at the interface when $T / T_{0}=1.01$ and $n / n_{0}=1.01$.

\begin{tabular}{cccccc}
\hline & & \multicolumn{2}{c}{$u$} \\
\cline { 3 - 5 }$\sigma=0.1$ & $\delta$ & linear & non-linear & linear & non-linear \\
\cline { 2 - 5 } & 0.1 & $4.268 \times 10^{-4}$ & $4.284 \times 10^{-4}$ & $5.362 \times 10^{-3}$ & $5.364 \times 10^{-3}$ \\
& 1 & $4.614 \times 10^{-4}$ & $4.617 \times 10^{-4}$ & $4.603 \times 10^{-3}$ & $4.679 \times 10^{-3}$ \\
& 10 & $5.573 \times 10^{-4}$ & $5.626 \times 10^{-4}$ & $1.510 \times 10^{-3}$ & $1.549 \times 10^{-3}$ \\
\hline$\sigma=0.5$ & 0.1 & $2.152 \times 10^{-3}$ & $2.158 \times 10^{-3}$ & $4.867 \times 10^{-3}$ & $4.523 \times 10^{-3}$ \\
& 1 & $2.454 \times 10^{-3}$ & $2.453 \times 10^{-3}$ & $3.795 \times 10^{-3}$ & $3.889 \times 10^{-3}$ \\
& 10 & $3.242 \times 10^{-3}$ & $3.265 \times 10^{-3}$ & $1.277 \times 10^{-3}$ & $1.293 \times 10^{-3}$ \\
\hline$\sigma=1$. & 0.1 & $4.360 \times 10^{-3}$ & $4.356 \times 10^{-3}$ & $3.403 \times 10^{-3}$ & $3.446 \times 10^{-3}$ \\
& 1 & $5.339 \times 10^{-3}$ & $5.323 \times 10^{-3}$ & $2.679 \times 10^{-3}$ & $2.746 \times 10^{-3}$ \\
& 10 & $8.157 \times 10^{-3}$ & $8.169 \times 10^{-3}$ & $8.500 \times 10^{-4}$ & $8.557 \times 10^{-4}$ \\
\hline
\end{tabular}

Table 9: Comparison between linear and non-linear results for the bulk velocity and heat flux at the interface when $T / T_{0}=1.01$ and $n / n_{0}=1.1$.

\begin{tabular}{|c|c|c|c|c|c|}
\hline \multirow{5}{*}{$\sigma=0.1$} & \multirow[b]{2}{*}{$\delta$} & \multicolumn{2}{|c|}{$u$} & \multicolumn{2}{|c|}{$q$} \\
\hline & & linear & non-linear & linear & non-linear \\
\hline & 0.1 & $2.971 \times 10^{-3}$ & $2.977 \times 10^{-3}$ & $4.112 \times 10^{-3}$ & $4.123 \times 10^{-3}$ \\
\hline & 1 & $3.039 \times 10^{-3}$ & $3.050 \times 10^{-3}$ & $3.597 \times 10^{-3}$ & $3.615 \times 10^{-3}$ \\
\hline & 10 & $3.185 \times 10^{-3}$ & $3.214 \times 10^{-3}$ & $1.281 \times 10^{-3}$ & $1.339 \times 10^{-3}$ \\
\hline \multirow[t]{3}{*}{$\sigma=0.5$} & 0.1 & $1.500 \times 10^{-2}$ & $1.474 \times 10^{-2}$ & $-1.823 \times 10^{-3}$ & $-1.719 \times 10^{-3}$ \\
\hline & 1 & $1.617 \times 10^{-2}$ & $1.596 \times 10^{-2}$ & $-1.515 \times 10^{-3}$ & $-1.459 \times 10^{-3}$ \\
\hline & 10 & $1.858 \times 10^{-2}$ & $1.843 \times 10^{-2}$ & $-5.491 \times 10^{-5}$ & $4.473 \times 10^{-5}$ \\
\hline \multirow[t]{3}{*}{$\sigma=1}$. & 0.1 & $3.040 \times 10^{-2}$ & $2.911 \times 10^{-2}$ & $-9.379 \times 10^{-3}$ & $-9.147 \times 10^{-3}$ \\
\hline & 1 & $3.518 \times 10^{-2}$ & $3.380 \times 10^{-2}$ & $-8.874 \times 10^{-3}$ & $-8.562 \times 10^{-3}$ \\
\hline & 10 & $4.664 \times 10^{-2}$ & $4.493 \times 10^{-2}$ & $-2.501 \times 10^{-3}$ & $-1.542 \times 10^{-3}$ \\
\hline
\end{tabular}


Table 10: Comparison between linear and non-linear results for the bulk velocity and heat flux at the interface when $T / T_{0}=1.05$ and $n / n_{0}=1.5$.

\begin{tabular}{|c|c|c|c|c|c|}
\hline \multirow{5}{*}{$\sigma=0.1$} & \multirow[b]{2}{*}{$\delta$} & \multicolumn{2}{|c|}{$u$} & \multicolumn{2}{|c|}{$q$} \\
\hline & & linear & non-linear & linear & non-linear \\
\hline & 0.1 & $1.486 \times 10^{-2}$ & $1.501 \times 10^{-2}$ & $2.056 \times 10^{-2}$ & $2.088 \times 10^{-2}$ \\
\hline & 1 & $1.519 \times 10^{-2}$ & $1.552 \times 10^{-2}$ & $1.799 \times 10^{-2}$ & $1.803 \times 10^{-2}$ \\
\hline & 10 & $1.593 \times 10^{-2}$ & $1.669 \times 10^{-2}$ & $6.407 \times 10^{-3}$ & $6.394 \times 10^{-3}$ \\
\hline$\sigma=0.5$ & 0.1 & $7.502 \times 10^{-2}$ & $6.889 \times 10^{-2}$ & $-9.116 \times 10^{-3}$ & $-6.904 \times 10^{-3}$ \\
\hline & 1 & $8.086 \times 10^{-2}$ & $7.594 \times 10^{-2}$ & $-7.576 \times 10^{-3}$ & $-6.069 \times 10^{-3}$ \\
\hline & 10 & $9.270 \times 10^{-2}$ & $9.037 \times 10^{-2}$ & $-2.745 \times 10^{-4}$ & $1.414 \times 10^{-3}$ \\
\hline & 0.1 & $1.520 \times 10^{-1}$ & $1.125 \times 10^{-1}$ & $-4.690 \times 10^{-2}$ & $-3.977 \times 10^{-2}$ \\
\hline & 1 & $1.759 \times 10^{-1}$ & $1.472 \times 10^{-1}$ & $-4.437 \times 10^{-2}$ & $-3.376 \times 10^{-1}$ \\
\hline & 10 & $2.332 \times 10^{-1}$ & $1.972 \times 10^{-1}$ & $-1.250 \times 10^{-2}$ & $9.454 \times 10^{-3}$ \\
\hline
\end{tabular}

